\title{
Long cycles in hypercubes with optimal number of faulty vertices
}

\author{
Jiří Fink * \\ Department of Applied Mathematics \\ Faculty of Mathematics and Physics \\ Charles University in Prague \\ e-mail: fink@kam.mff.cuni.cz
}

\author{
Petr Gregor ${ }^{\dagger}$ \\ Department of Theoretical Computer \\ Science and Mathematical Logic \\ Charles University in Prague \\ e-mail: gregor@ktiml.mff.cuni.cz
}

\begin{abstract}
Let $f(n)$ be the maximum integer such that for every set $F$ of at most $f(n)$ vertices of the hypercube $Q_{n}$, there exists a cycle of length at least $2^{n}-2|F|$ in $Q_{n}-F$. Castañeda and Gotchev conjectured that $f(n)=\left(\begin{array}{c}n \\ 2\end{array}\right)-2$. We prove this conjecture. We also prove that for every set $F$ of at most $\left(n^{2}+n-4\right) / 4$ vertices of $Q_{n}$, there exists a path of length at least $2^{n}-2|F|-2$ in $Q_{n}-F$ between any two vertices such that each of them has at most 3 neighbors in $F$. We introduce a new technique of potentials which could be of independent interest.
\end{abstract}

\section{Introduction}

The $n$-dimensional hypercube $Q_{n}$ is the (bipartite) graph with all binary vectors of length $n$ as vertices and edges joining every two vertices that differ in exactly one coordinate. The bipartite classes of $Q_{n}$ consist of vertices with even, respectively odd, weight, where the weight $|u|$ of a vertex $u \in V\left(Q_{n}\right)=\{0,1\}^{n}$ is defined as the number of 1's in $u$. A set $F \subseteq V\left(Q_{n}\right)$ in which all vertices are from the same bipartite class, is called a monopartite set.

Applications of the hypercube in the theory of interconnection networks inspired many questions related to its robustness. In particular, if some faulty (or busy) vertices $F \subseteq V\left(Q_{n}\right)$ and all incident edges are removed from $Q_{n}$, is there a cycle in the remaining graph, denoted by $Q_{n}-F$, which covers 'almost' all vertices? And how many vertices in the worst-case can be removed?

Clearly, if $F$ is monopartite, the length of any cycle in $Q_{n}-F$ cannot exceed $2^{n}-2|F|$. This leads to the following definition. A cycle of length at least $2^{n}-2|F|$ in $Q_{n}-F$ is called a long $F$-free cycle in $Q_{n}$. Let $f(n)$ be the maximum integer such that $Q_{n}-F$ has a long $F$-free cycle for every set $F$ of at most $f(n)$ vertices in $Q_{n}$.

The study of this parameter has a numerous literature. Firstly, Chan and Lee [2] showed that $f(n) \geq(n-1) / 2$. Then, Yang et al. [15] improved it to $f(n) \geq n-2$, and Tseng et al. [13] to $f(n) \geq n-1$. Next, Fu [7] significantly increased it to $f(n) \geq 2 n-4$ for $n \geq 3$, and

${ }^{*}$ The Institute for Theoretical Computer Science (ITI) is supported by project 1M0545 of the Czech Ministry of Education.

${ }^{\dagger}$ Partially supported by the Czech Science Foundation Grant 201/08/P298. 
Castañeda and Gotchev [1] strengthened it further to $f(n) \geq 3 n-7$ for $n \geq 5$. Recently, Fink and Gregor [6] obtained the first quadratic lower bound $f(n) \geq n^{2} / 10+n / 2+1$ for $n \geq 15$.

On the other hand, Koubek [10] and independently Castañeda and Gotchev [1] noticed that for every $n \geq 4$ there is a set $F$ of $\left(\begin{array}{l}n \\ 2\end{array}\right)-1$ vertices such that $Q_{n}-F$ contains no cycle of length at least $2^{n}-2|F|$, so $f(n) \leq\left(\begin{array}{l}n \\ 2\end{array}\right)-2$. An example of a such set $F$ consists of all but one vertex of weight 2 . Indeed, since all vertices of $F$ have even weight, any long $F$-free cycle in $Q_{n}$ must visit all the remaining vertices of even weight. Namely, it has to visit the vertex $\mathbf{0}=(0, \ldots, 0)$ and some vertex of weight 4 , which is clearly impossible as they are in different 2-connected components of $Q_{n}-F$.

From the previous results it follows that the above upper bound is sharp for $n=4$ [7] and for $n=5$ [1]. It was conjectured [1] that it is sharp for all $n \geq 4$, i.e. $f(n)=\left(\begin{array}{l}n \\ 2\end{array}\right)-2$ for $n \geq 4$. In this paper we prove this conjecture.

Theorem 1.1. For every set $F$ of at most $\left(\begin{array}{l}n \\ 2\end{array}\right)-2$ vertices in $Q_{n}$ and $n \geq 4$, the graph $Q_{n}-F$ contains a cycle of length at least $2^{n}-2|F|$.

To prove Theorem 1.1, we need to consider a modification of this problem for long paths with prescribed endvertices. Similarly as above, a path in $Q_{n}-F$ between vertices $u$ and $v$, and of length at least $2^{n}-2|F|-2$ is called a long $F$-free uv-path in $Q_{n}$. Note that in case $u$ and $v$ are from different bipartite classes, the length of any long $F$-free $u v$-path is at least $2^{n}-2|F|-1$. Also note that in the case when $F \cup\{u, v\}$ is monopartite, the length of any $u v$-path in $Q_{n}-F$ cannot exceed $2^{n}-2|F|-2$, and hence a long $F$-free $u v$-path has optimal length.

$\mathrm{Fu}[8]$ showed that $Q_{n}-F$ contains a long path between any two vertices if $|F| \leq n-2$ and $n \geq 3$. To improve this result for larger sets $F$, one needs to introduce additional conditions on the neighbors of prescribed endvertices. Kueng et al. [11] strengthened the number of tolerable faults to $|F| \leq 2 n-5$ under the condition that the minimal degree of $Q_{n}-F$ is at least 2. Recently, Fink and Gregor [6] showed that a much weaker condition is both necessary and sufficient for sets $F$ with $|F| \leq 2 n-4$. Let $N(u)$ be the set of neighbors of a vertex $u$ in $Q_{n}$.

Theorem 1.2 (Fink and Gregor [6]). Let $F$ be a set of at most $2 n-4$ faulty vertices of $Q_{n}$ and $n \geq 5$. For every two vertices $u$ and $v$ of $Q_{n}-F$, there exists a long $F$-free uv-path in $Q_{n}$ if and only if $N(u) \nsubseteq F \cup\{v\}$ and $N(v) \nsubseteq \nsubseteq F \cup\{u\}$.

Note that for $|F| \leq n-2$, the right side of the equivalence in Theorem 1.2 is always satisfied. Hence, we obtain the following direct corollary.

Corollary $1.3(\mathrm{Fu}[8])$. For every set $F$ of at most $n-2$ vertices of $Q_{n}$ and $n \geq 2$, there is a long $F$-free uv-path in $Q_{n}$ between every two vertices $u$ and $v$ of $Q_{n}-F$.

In this paper, we show that $F$ can be as large as $f(n+1) / 2$ if both prescribed endvertices have only few neighbors in $F$.

Theorem 1.4. For every set $F$ of at most $\left(n^{2}+n-4\right) / 4$ vertices in $Q_{n}$ and $n \geq 5$, the graph $Q_{n}-F$ contains a path of length at least $2^{n}-2|F|-2$ between every two vertices such that each of them has at most 3 neighbors in $F$.

The general difficulty with quadratic bounds on $|F|$ in Theorems 1.1 and 1.4 is that the hypercube cannot be always split into subcubes so that the bounds hold in each subcube. 
Thus, the standard induction technique fails. We introduce up to our knowledge a new technique of so called potentials which allows us to effectively deal with such situations.

Furthermore, in the proof of Theorem 1.4 we need to consider the following extension of the studied problem for two paths. Assume that we have two different (but not necessarily disjoint) sets $A=\{u, v\}$ and $B=\{x, y\}$ of vertices of $Q_{n}-F$. A path $P$ between a vertex of $A$ and a vertex of $B$ is called an $A B$-path. Its length $|P|$ is the number of edges in $P$. A pair $P_{1}, P_{2}$ of vertex-disjoint $A B$-paths in $Q_{n}-F$ is called an $F$-free $A B$-routing in $Q_{n}$. Moreover, it is said to be long if $\left|P_{1}\right|+\left|P_{2}\right| \geq 2^{n}-2|F|-3$. Note that if $A$ and $B$ are not disjoint, say $A \cap B=\{u=x\}$, then any long $F$-free $A B$-routing consists of the $u u$-path of length 0 and an $v y$-path of length at least $2^{n}-2|F|-3$.

We studied those problems separately in [5] where we obtained the following results. ${ }^{1}$

Theorem 1.5 ([5]). For every set $F$ of at most $n-3$ vertices in $Q_{n}$ and $n \geq 4$, there exists a long $F$-free $A B$-routing in $Q_{n}$ between every two different sets $A, B \subseteq V\left(Q_{n}\right) \backslash F$ such that $|A|=|B|=2$ and $A \cup B$ is not monopartite.

As a consequence, if $F \cup\{u, v\}$ is not monopartite, we obtain an $u v$-path in $Q_{n}-F$ of length at least $2^{n}-2|F|-1$, which is more than is guaranteed by long paths.

Corollary 1.6 ([5]). For every set $F$ of at most $n-2$ vertices of $Q_{n}$ and $n \geq 4$, the graph $Q_{n}-F$ has an uv-path of length at least $2^{n}-2|F|-1$ for every two vertices $u, v \in V\left(Q_{n}\right) \backslash F$ such that $F \cup\{u, v\}$ is not monopartite.

From Theorem 1.1 it follows that the decision problem whether the hypercube $Q_{n}$ for the given set $F$ of faulty vertices contains an $F$-free cycle has a trivial answer if $|F| \leq\left(\begin{array}{l}n \\ 2\end{array}\right)-2$. On the other hand, Dvořák and Koubek [4] showed that this problem is NP-hard if $|F|$ is unbounded. Moreover, they [4] presented a function $\phi(n)=\Theta\left(n^{6}\right)$ such that the problem remains NP-hard even if $|F| \leq \phi(n)$. Furthermore, Dvořák and Koubek [3] described a polynomial algorithm for the similar decision problem of long $F$-free paths between given vertices in $Q_{n}$ if $|F| \leq n^{2} / 10+n / 2+1$.

For the completeness, let us also mention that there are many related results on similar problems of bipanconnectivity, bipancyclicity, long cycles, and long paths in various modifications of faulty hypercubes, see a survey of Xu and Ma [14] for further references.

\section{Preliminaries}

The $n$-dimensional hypercube $Q_{n}$ is the (bipartite) graph with all binary vectors of length $n$ as vertices and edges joining every two vertices that differ in exactly one coordinate. Let $\mathbf{0}$ denote the vertex of $Q_{n}$ consisting of all 0's. For every $i \in[n]=\{1,2, \ldots, n\}$ let $e_{i}$ denote the vertex with 1 exactly in the $i$-th coordinate. Furthermore, for every distinct $i, j \in[n]$ let $e_{i, j}$ denote the vertex with 1 exactly in the $i$-th and $j$-th coordinate.

Let $d(u, v)$ be the (Hamming) distance of vertices $u$ and $v$ in $Q_{n}$, i.e. the number of coordinates where $u$ and $v$ differ. Recall that the weight $|u|$ of a vertex $u$ is the number of 1 's in $u$, i.e. $|u|=d(u, \mathbf{0})$. The vertices of even and odd weight, respectively, form bipartite classes of $Q_{n}$. The parity of a vertex $u$ is the parity of its weight $|u|$. Hence, two vertices have the same parity if and only if they are in the same bipartite class. The $k$-th level of $Q_{n}$ is the set of vertices of weight $k$ for $0 \leq k \leq n$.

\footnotetext{
${ }^{1}$ This paper has not been published yet, so we include the proofs for the purpose of referee in the appendix.
} 
Clearly, $Q_{n}$ has a regular degree $n$. Let $N(u)$ be the set of neighbors of a vertex $u$ in $Q_{n}$, and let $N^{+}(u)$ and $N^{-}(u)$ be the sets neighbors of $u$ with weight $|u|+1$ and $|u|-1$, respectively. It is well-known that every two vertices of $Q_{n}$ have 0 or 2 common neighbors.

In order to apply induction, we need to split the hypercube $Q_{n}$ into two $(n-1)$-dimensional subcubes $Q_{i: L}$ and $Q_{i: R}$. This is obtained by fixing some coordinate $i \in[n]$. Formally, we define the subcube $Q_{i: L}$ as the subgraph of $Q_{n}$ induced by vertices that have 0 on the $i$-th coordinate. Similarly, the subcube $Q_{i: R}$ is the subgraph of $Q_{n}$ induced by vertices that have 1 on the $i$-th coordinate. For a vertex $x$ of $Q_{i: L}$, let $x_{R}$ be the (only) neighbor of $x$ in $Q_{i: R}$. Similarly for a vertex $x$ of $Q_{i: R}$, let $x_{L}$ be the (only) neighbor of $x$ in $Q_{i: L}$.

Assume that $F$ is a given set of faulty vertices of $Q_{n}$. The vertices of $Q_{n}$ which are not in $F$ are called $F$-free. For every $i \in[n]$ we define $F_{i: L}$ and $F_{i: R}$ to be the sets of faulty vertices in $Q_{i: L}$ and $Q_{i: R}$, respectively. Let $F^{k}$ be the set of vertices of $F$ from level $k$ (i.e. of weight $k$ ) for $0 \leq k \leq n$. Similarly, let $F^{\geq k}$ be the set of vertices of $F$ from level at least $k$. Furthermore, we define $F_{i: L}^{k}=F^{k} \cap F_{i: L}$ and $F_{i: R}^{k}=F^{k} \cap F_{i: R}$. For a vertex $u$ of $Q_{n}$ let $F(u)$ be the set of faulty neighbors of $u$, i.e. $F(u)=F \cap N(u)$.

Let $A_{F}$ be the $|F| \times n$ matrix whose rows are the binary vectors representing the vertices of $F$. Let $\left|A_{F}\right|$ be the number of ones in $A_{F}$. Clearly, $\left|A_{F}\right|$ is the sum of $|x|$ over all $x \in F$. Note that $\left|F_{i: L}\right|$ and $\left|F_{i: R}\right|$ are the numbers of zeros and ones, respectively, in the $i$-th column of $A_{F}$. By symmetry of $Q_{n}$, we assume that

$$
\left|F_{i: L}\right| \geq\left|F_{i: R}\right| \text { for every dimension } i \in[n] .
$$

Indeed, by exchanging zeros and ones in those columns $i \in[n]$ where $\left|F_{i: L}\right|<\left|F_{i: R}\right|$ we obtain an automorphism of $Q_{n}$ that maps the set $F$ to a new set satisfying the condition (1).

To apply Theorem 1.2 we need to bound the number $\alpha(F)$ of vertices of $Q_{n}$ that have at least 4 neighbors in $F$.

Proposition 2.1. For every set $F \subseteq V\left(Q_{n}\right)$ it holds that

$$
\alpha(F) \leq \min \left\{\frac{n|F|}{4}, \frac{\left(\begin{array}{c}
|F| \\
2
\end{array}\right)}{3}\right\} .
$$

Proof. Every vertex from $F$ has $n$ neighbors in $Q_{n}$, but every vertex $x$ with $|F(x)| \geq 4$ has at least 4 neighbors in $F$. Hence, $\alpha(F) \leq n|F| / 4$.

In order to prove the second inequality of this proposition we compute the number $p$ of pairs of incident edges $u x$ and $v x$ of $Q_{n}$ where $u, v \in F$ are distinct neighbors of $x$. Since every two vertices $u$ and $v$ of $Q_{n}$ have at most 2 neighbors in common, we have $p \leq 2\left(\begin{array}{c}|F| \\ 2\end{array}\right)$. On the other hand, every vertex $x$ with $|F(x)| \geq 4$ has at least $\left(\begin{array}{l}4 \\ 2\end{array}\right)=6$ pairs of vertices from $F$ in its neighborhood, so $6 \alpha(F) \leq p$. Hence, $\alpha(F) \leq\left(\begin{array}{c}|F| \\ 2\end{array}\right) / 3$.

Proposition 2.2. For every set $F \subseteq V\left(Q_{n}\right)$ with $|F| \leq 6$ it holds that $\alpha(F) \leq 2$.

Proof. Suppose for a contradiction that there exist three vertices $a, b$ and $c$ in $Q_{n}$ such that $|F(a)|,|F(b)|,|F(c)| \geq 4$. Without lost of generality we assume that $a=\mathbf{0}$. Hence, there are at least 4 faulty vertices in the first level. Since there remain at most two vertices in $F \backslash F(a)$, the vertices $b$ and $c$ both share exactly 2 faulty neighbors with the vertex $a$, so they are in the second level. Furthermore, it follows that the vertices $b$ and $c$ share two neighbors $x, y \in F^{3}$, so $(b, x, c, y)$ forms a cycle of length 4 . But this contradicts the structure of $Q_{n}$ since every cycle of length 4 in $Q_{n}$ is contained in exactly 3 consecutive levels. 


\section{Overview of the proofs}

In this section we give an overview of main proofs and explain the general ideas.

The proofs of Theorems 1.1 and 1.4 have very similar structure. In both theorems we are given a set of faulty vertices $F$ in $Q_{n}$, but the maximal cardinality of $F$ differs. For general purposes, let us denote the maximal cardinality of $F$ by $z(n)$. In Theorem 1.1 we have $z(n)=\left(\begin{array}{l}n \\ 2\end{array}\right)-2$, and in Theorem 1.4 we have $z(n)=\left\lfloor\frac{n^{2}+n-4}{4}\right\rfloor$.

Both proofs proceed by induction on the dimension $n$. Fortunately, the base of induction for $n=5$ is already known in both cases. For Theorem 1.1 it directly follows from the following result.

Theorem 3.1 (Castañeda and Gotchev [1]). For every set $F$ of at most $3 n-7$ vertices in $Q_{n}$ and $n \geq 5$, the graph $Q_{n}-F$ contains a cycle of length at least $2^{n}-2|F|$.

For Theorem 1.4, the base of induction follows from Theorem 1.2 since $2 n-4=\left\lfloor\frac{n^{2}+n-4}{4}\right\rfloor$ for $n=5$, and the condition that $|F(u)|,|F(v)| \leq 3$ implies that $N(u) \nsubseteq F \cup\{v\}$ and $N(v) \not F \cup\{u\}$ for $n=5$.

Hence, our task remains to prove the induction step for both Theorems 1.1 and 1.4. Although they are applied in the proofs of each other, note that it is done in a correct way, since the induction steps proceed together. That is, the statements of Theorem 1.1 and Theorem 1.4 for $n$ requires only that

$$
\text { the statements of Theorem } 1.1 \text { and Theorem } 1.4 \text { hold for } n-1 \text {. }
$$

In the first part of the induction steps we assume that

$$
\text { there exists a dimension } i \in[n] \text { such that }\left|F_{i: L}\right|,\left|F_{i: R}\right| \leq z(n-1) \text {. }
$$

In this case in Theorem 1.4 we proceed directly by applying induction (2) on both $Q_{i: L}$ and $Q_{i: R}$. In Theorem 1.1 we obtain from (1) that ${ }^{2}$

$$
\left|F_{i: R}\right| \leq\left\lfloor\frac{|F|}{2}\right\rfloor \leq\left\lfloor\frac{\left(\begin{array}{l}
n \\
2
\end{array}\right)-2}{2}\right\rfloor=\left\lfloor\frac{(n-1)^{2}+(n-1)-4}{4}\right\rfloor .
$$

Therefore, we may directly apply induction (2): Theorem 1.1 in $Q_{i: L}$ and Theorem 1.4 in $Q_{i: R}$.

\subsection{Potentials}

In the second part of both proofs we assume that (3) does not hold. The assumption (1) implies that

$$
\left|F_{i: L}\right|>z(n-1) \text { for every dimension } i \in[n] .
$$

Now we introduce up to our knowledge a new method of so called potentials which is used in the both proofs of Theorems 1.1 and 1.4.

Let $k(n)=z(n)-z(n-1)-1$. Note that if (4) holds, then $\left|F_{i: R}\right|=|F|-\left|F_{i: L}\right| \leq k(n)$ for every dimension $i \in[n]$. We define the potentials of the set $F$ as follows:

\footnotetext{
${ }^{2}$ This explains why we consider at most $\left\lfloor\frac{n^{2}+n-4}{4}\right\rfloor$ faulty vertices in Theorem 1.4.
} 
- $\phi_{0}(F)=2\left(1-\left|F^{0}\right|\right)= \begin{cases}0 & \text { if } \mathbf{0} \in F \\ 2 & \text { if } \mathbf{0} \notin F,\end{cases}$

- $\phi_{1}(F)$ is the number of $F$-free vertices in the first level, i.e. $\phi_{1}(F)=n-\left|F^{1}\right|$,

- $\phi_{\geq 3}(F)$ is the sum of $|x|-2$ over all faulty vertices $x$ in level at least 3,

- $\phi_{\operatorname{dim}}(F)$ is the sum of $\left|F_{i: L}\right|-z(n-1)-1$ over all dimensions $i \in[n]$,

- $\phi(F)=\phi_{0}(F)+\phi_{1}(F)+\phi_{\geq 3}(F)+\phi_{\text {dim }}(F)$.

Clearly, $\phi_{0}(F), \phi_{1}(F), \phi_{\geq 3}(F)$ are non-negative. Furthermore, it follows from (4) that $\phi_{\text {dim }}(F)$ is non-negative. Consequently, $\phi(F)$ is non-negative.

Intuitively, the potential $\phi_{0}(F)+\phi_{1}(F)+\phi_{\geq 3}(F)$ determines how much the set $F$ differs from a set $F^{\prime}$ with a minimal number of ones in the matrix $A_{F^{\prime}}$. If $\mathbf{0} \notin F$, we pay by $\phi_{0}(F)=2$; otherwise, $\phi_{0}(F)=0$. For every vertex of weight 1 which is not in $F$, we pay by 1 in $\phi_{1}(F)$. For every vertex of $F$ which has weight at least 3 , we pay its distance to the second level in $\phi_{\geq 3}(F)$. Finally, for every dimension $i \in[n]$ we know that $\left|F_{i: L}\right|>z(n-1)$ since we assume (4), therefore we pay in $\phi_{\operatorname{dim}}(F)$ the number of vertices which could be moved from $F_{i: L}$ to $F_{i: R}$ so that (4) remains satisfied.

Observe that the definition of $\phi_{\operatorname{dim}}(F)$ and (4) implies that if $\phi_{\operatorname{dim}}(F)<n$, then there exists a dimension $i \in[n]$ such that $\left|F_{i: L}\right|=z(n-1)+1$. Now, we compute the potential $\phi(F)$ of the set $F$. Note that the potential $\phi(F)$ depends only on $|F|, z(n)$ and $z(n-1)$.

Proposition 3.2. If $|F| \leq z(n)$ and $\left|F_{i: L}\right|>z(n-1)$ for every dimension $i \in[n]$, then

$$
\phi(F)=n k(n)-2 z(n)+n+2-(n-2)(z(n)-|F|) .
$$

Proof. We prove the requested equality by double-counting the number of 1's in the matrix $A_{F}$. First, we sum up 1's by columns. Since

$$
\left|F_{i: R}\right|=|F|-\left|F_{i: L}\right|=k(n)-(z(n)-|F|)-\left(\left|F_{i: L}\right|-z(n-1)-1\right),
$$

we have

$$
\left|A_{F}\right|=\sum_{i \in[n]}\left|F_{i: R}\right|=n k(n)-n(z(n)-|F|)-\phi_{\operatorname{dim}}(F) .
$$

Now, we sum up 1's by rows.

$$
\begin{aligned}
\left|A_{F}\right| & =\sum_{x \in F}|x|=0\left|F^{0}\right|+1\left|F^{1}\right|+2\left|F^{2}\right|+2\left|F^{\geq 3}\right|+\phi_{\geq 3}(F) \\
& =\phi_{0}(F)+\phi_{1}(F)+\phi_{\geq 3}(F)+2|F|-n-2 .
\end{aligned}
$$

The requested equality follows.

Let us explain informally how potentials are useful for us. Below in Proposition 4.2 we compute the particular value of $\phi(F)$ for paths when $z(n)=\left\lfloor\frac{n^{2}+n-4}{4}\right\rfloor$; and in Proposition 5.2 we compute it for cycles when $z(n)=\left(\begin{array}{l}n \\ 2\end{array}\right)-2$. We will see that $\phi(F)$ is small in both cases. This allows us to split $Q_{n}$ into $Q_{i: L}$ and $Q_{i: R}$ so that $\left|F_{i: L}\right|=z(n-1)+1$, i.e. there is one faulty vertex more in $F_{i: L}$ than is allowed for applying induction. In such situations 
we ignore one properly chosen vertex $x \in F_{i: L}$ and try to proceed directly. If the vertex $x$ belongs to the obtained path (or cycle), we attempt to detour it.

However, those detours may also fail because of another vertex $y \in F_{i: R}$. Nevertheless, if this happens, the vertex $y$ must contribute into $\phi_{\geq 3}(F)$. By combination of those methods we either find a long $F$-free path in $Q_{n}$ or obtain a contradiction with a small potential $\phi(F)$.

\section{Long paths}

In this section we prove Theorem 1.4. In what follows assume that $F$ is a set of at most $z(n)=\left\lfloor\frac{n^{2}+n-4}{4}\right\rfloor$ vertices of $Q_{n}, n \geq 5$, and $u, v$ are distinct vertices of $Q_{n}-F$ with $|F(u)|,|F(v)| \leq 3$. Recall that Theorem 1.4 says that $Q_{n}-F$ contains a path between $u$ and $v$ of length at least $2^{n}-2|F|-2$. Such path is called a long $F$-free $u v$-path.

The proof proceeds by induction on the dimension $n$. For $n=5$ the statement follows from Theorem 1.2 since $|F| \leq z(5)=6$. Now, we prove the induction step for $n \geq 6$. We divide the proof into two main parts.

\subsection{Induction-friendly split}

In the first part, we consider the case when $Q_{n}$ can be split into $Q_{i: L}$ and $Q_{i: R}$ by a dimension $i \in[n]$ such that $\left|F_{i: L}\right|,\left|F_{i: R}\right| \leq z(n-1)$; see (3). In this case, we apply induction directly.

Lemma 4.1. Let $Q_{n}$ be split into subcubes $Q_{i: L}$ and $Q_{i: R}$ so that $\left|F_{i: L}\right|,\left|F_{i: R}\right| \leq z(n-1)$. Then there exists a long $F$-free uv-path $P$ in $Q_{n}$. Moreover, if $\left|F_{i: L}^{1}\right| \geq n-2$, then $\mathbf{0} \notin P$.

Proof. Since the dimension $i$ is fixed, in this proof we omit the index $i$ to simplify the notation. We distinguish two cases regarding the position of vertices $u$ and $v$ in $Q_{L}$ and $Q_{R}$.

Case 1: If $u, v$ are in different subcubes, say $u \in V\left(Q_{L}\right)$ and $v \in V\left(Q_{R}\right)$, then our aim is to find a vertex $x$ in $Q_{L}$ of opposite parity to the parity of $u$ such that $x \notin F_{L}, x_{R} \notin F_{R} \cup\{v\}$ and $\left|F_{L}(x)\right|,\left|F_{R}\left(x_{R}\right)\right| \leq 3$. If there is a such vertex $x$, then by induction (2), $Q_{L}$ has a long $F_{L}$-free $u x$-path $P_{L}$ of length at least $2^{n-1}-2\left|F_{L}\right|-1, Q_{R}$ has a long $F_{R}$-free $x_{R} v$-path $P_{R}$ of length at least $2^{n-1}-2\left|F_{R}\right|-2$. Hence, their concatenation by the edge $x x_{R}$ is the requested long $F$-free $u v$-path $P$ in $Q_{n}$ since

$$
|P|=\left|P_{L}\right|+\left|P_{R}\right|+1 \geq 2^{n-1}-2\left|F_{L}\right|-1+2^{n-1}-2\left|F_{R}\right|-2+1=2^{n}-2|F|-2 .
$$

Let $A$ be the set of $2^{n-2}$ vertices $x$ in $Q_{L}$ with the opposite parity to the parity of $u$. We count for how many vertices $x$ from $A$ at least one of the following conditions fails: $x \notin F_{L}$, $x_{R} \notin F_{R} \cup\{v\}$, and $\left|F_{L}(x)\right|,\left|F_{R}\left(x_{R}\right)\right| \leq 3$. First, we find an upper bound on the number of vertices from $A$ such that $x \in F_{L}$ or $\left|F_{L}(x)\right| \geq 4$.

Every vertex of $F_{L} \backslash A$ has $n-1$ neighbors in $A$, so there are at most $\frac{n-1}{4}\left|F_{L} \backslash A\right|$ vertices $x$ of $A$ such that $\left|F_{L}(x)\right| \geq 4$. Furthermore, we have $\left|F_{L} \cap A\right|$ vertices in $A$ such that $x \in F_{L}$. Thus, the number of vertices $x$ of $A$ such that $x \in F_{L}$ or $\left|F_{L}(x)\right| \geq 4$ is at most

$$
\frac{n-1}{4}\left|F_{L} \backslash A\right|+\left|F_{L} \cap A\right| \leq \frac{n-1}{4}\left|F_{L}\right|
$$

since $n \geq 6$.

Similarly, the number of vertices $x$ of $A$ such that $x_{R} \in F_{R}$ or $\left|F_{R}\left(x_{R}\right)\right| \geq 4$ is at most $\frac{n-1}{4}\left|F_{R}\right|$. Finally, at most one vertex $x$ of $A$ has $x_{R}=v$. Altogether, we have at 
most $\frac{n-1}{4}|F|+1 \leq \frac{n-1}{4} z(n)+1$ vertices $x$ in $A$ such that $x \notin F_{L}, x_{R} \notin F_{R} \cup\{v\}$, and $\left|F_{L}(x)\right|,\left|F_{R}\left(x_{R}\right)\right| \leq 3$, which is less than $|A|=2^{n-2}$ for $n \geq 6$. Therefore, the desired vertex $x$ exists.

Case 2: If $u, v$ are in the same subcube, say $u, v \in V\left(Q_{L}\right)$, then by induction (2), there exists a long $F_{L}$-free $u v$-path $P_{L}$ in $Q_{L}$. Our aim is to find an edge $x y$ of $P_{L}$ such that $x_{R}, y_{R} \notin F_{R}$ and $\left|F_{R}\left(x_{R}\right)\right|,\left|F_{R}\left(y_{R}\right)\right| \leq 3$. If there is such edge $x y$, then by induction, $Q_{R}$ contains a long $F_{R}$-free $x_{R} y_{R}$-path $P_{R}$. By replacing the edge $x y$ in $P_{L}$ with the path $\left(x, P_{R}, y\right)$, we obtain the requested long $F$-free $u v$-path $P$ in $Q_{n}$ since

$$
|P|=\left|P_{L}\right|+\left|P_{R}\right|+1 \geq 2^{n-1}-2\left|F_{L}\right|-2+2^{n-1}-2\left|F_{R}\right|-1+1=2^{n}-2|F|-2 .
$$

The path $P_{L}$ has at least $2^{n-1}-2\left|F_{L}\right|-2$ edges. Every vertex $z$ in $Q_{R}$ such that $z \in F_{R}$ or $\left|F_{R}(z)\right| \geq 4$ can block at most two edges $x y$ of $P_{L}$. We find an upper bound on the number of such vertices $z$.

By Proposition 2.1, there are $\alpha\left(F_{R}\right) \leq \min \left\{\frac{n-1}{4}\left|F_{R}\right|,\left(\begin{array}{c}\left|F_{R}\right| \\ 2\end{array}\right) / 3\right\}$ vertices $z$ in $Q_{R}$ such that $\left|F_{R}(z)\right| \geq 4$. Hence, the number of edges $x y$ of $P_{L}$ such that $x_{R}, y_{R} \notin F_{R}$ and $\left|F_{R}\left(x_{R}\right)\right|,\left|F_{R}\left(y_{R}\right)\right| \leq 3$ is at least

$\left|P_{L}\right|-2\left(\left|F_{R}\right|+\alpha\left(F_{R}\right)\right) \geq 2^{n-1}-2|F|-2 \alpha\left(F_{R}\right)-2 \geq\left\{\begin{array}{l}2^{n-1}-2 z(n)-2\left(\begin{array}{c}z(n-1) \\ 2\end{array}\right) / 3-2, \text { and } \\ 2^{n-1}-2 z(n)-\frac{n-1}{2} z(n-1)-2,\end{array}\right.$

which is positive for $n=6$ in the first case, and for $n \geq 7$ in the latter one. Therefore, the desired edge $x y$ exists.

It remains to prove the second part of the statement. Assume that $\left|F_{L}^{1}\right| \geq n-2$. Since the vertex $\mathbf{0}$ has $n-1$ neighbors in $Q_{L}$, at most one of them is $F_{L}$-free. Recall that each endvertex of the path $P_{L}$ has at most 3 neighbors in $F_{L}$ and $n \geq 6$. Hence, the path $P_{L}$ does not contain the vertex $\mathbf{0}$, and therefore also $\mathbf{0} \notin P$.

\subsection{Potentials}

In the second part of the proof of Theorem 1.4 we assume that (3) fails, i.e. (4) holds.

By substituting $z(n)=\left\lfloor\frac{n^{2}+n-4}{4}\right\rfloor$ and $k(n)=z(n)-z(n-1)-1$ into Proposition 3.2 we immediatelly obtain the following table of values of the potential $\phi(F)$ for $n=4 m+(n \bmod 4)$ where $m=\lfloor m / 4\rfloor$. Note that $k(n) \leq\left\lfloor\frac{n-1}{2}\right\rfloor$ in the all four cases.

\begin{tabular}{|c||c|c|c|}
\hline$n$ & $z(n)$ & $k(n)$ & $\phi(F)$ \\
\hline \hline $4 m$ & $4 m^{2}+m-1$ & $2 m-1$ & $4-2 m-(n-2)(z(n)-|F|)$ \\
\hline $4 m+1$ & $4 m^{2}+3 m-1$ & $2 m-1$ & $4-4 m-(n-2)(z(n)-|F|)$ \\
\hline $4 m+2$ & $4 m^{2}+5 m$ & $2 m$ & $4-2 m-(n-2)(z(n)-|F|)$ \\
\hline $4 m+3$ & $4 m^{2}+7 m+2$ & $2 m+1$ & $4-(n-2)(z(n)-|F|)$ \\
\hline
\end{tabular}

Lemma 4.2. If $\left|F_{i: L}\right|>z(n-1)$ for every dimension $i \in[n]$, then $|F|=z(n)$. Moreover, $\phi(F)=2$ for $n=6$ and $\phi(F) \leq 4$ for $n \geq 7$.

Proof. Since $\phi(F) \geq 0$ and $n \geq 6$, we have $(n-2)(z(n)-|F|)=0$ in the above table, so $|F|=z(n)$. The above table also implies the second part of this statement. 
In the rest of the proof we proceed by contradiction, so let us suppose that $F$ is a set of at most $z(n)$ vertices of $Q_{n}$ and $u, v$ are distinct vertices with $|F(u)|,|F(v)| \leq 3$ such that

$$
Q_{n} \text { does not contain a long } F \text {-free } u v \text {-path. }
$$

Recall that Lemma 4.1 implies that the assumption (3) fails. In the next lemma we consider the configurations when faulty vertex $\mathbf{0}$ has at most two $F$-free neighbors in $Q_{n}$.

Lemma 4.3. $0 \notin F$ or $\left|F^{1}\right| \leq n-2$.

Proof. For a contradiction, suppose $\mathbf{0} \in F$ and $\left|F^{1}\right| \geq n-1$. Since $n \geq 6$ and $\phi_{\operatorname{dim}}(F) \leq 4$ by Lemma 4.2 , there exists $i \in[n]$ such that $\left|F_{i: L}\right|=z(n-1)+1$. It follows that (3) holds for the set $F^{\prime}=F \backslash\{\mathbf{0}\}$ as $\mathbf{0} \in F_{i: L}$. Thus, there exists a long $F^{\prime}$-free $u v$-path $P$ in $Q_{n}$ by Lemma 4.1. Since $\left|F_{i: L}^{1}\right| \geq n-2$, Lemma 4.1 implies that the path $P$ does not contain the vertex $\mathbf{0}$. Therefore, $P$ is also a long $F$-free $u v$-path contrary to (5).

Corollary 4.4. $\phi_{\geq 3}(F) \leq 2$ for $n \geq 7$, and $\phi_{\geq 3}(F)=0$ for $n=6$.

Proof. Lemma 4.3 implies that $\phi_{0}(F)+\phi_{1}(F) \geq 2$. The rest follows from Lemma 4.2.

The following corollary shows that we can use Theorem 1.1 to find a long $F_{i: L}$-free cycle in $Q_{i: L}$ for every dimension $i \in[n]$.

Corollary 4.5. $\left|F_{i: L}\right| \leq\left(\begin{array}{c}n-1 \\ 2\end{array}\right)-2$ for every $i \in[n]$.

Proof. For a contradiction, suppose $\left|F_{i: L}\right|>\left(\begin{array}{c}n-1 \\ 2\end{array}\right)-2$ for some $i \in[n]$. Since $\left|F_{i: L}\right| \leq|F|=$ $z(n)=\left\lfloor\frac{n^{2}+n-4}{4}\right\rfloor$ and $n \geq 6$, the only possible values are $n=6$ and $\left|F_{i: L}\right|=z(6)=9$. Thus $\left|F_{i: R}\right|=0$, and consequently, $\phi_{\operatorname{dim}}(F) \geq 2$. But this contradicts $\phi(F)=2$ from Lemma 4.2 and $\phi_{0}(F)+\phi_{1}(F) \geq 2$ from Lemma 4.3.

Lemma 4.6. If $\phi_{\geq 3}(F) \geq 2$ or $n=6$, then $\left|F^{1}\right|=n$.

Proof. If $\phi_{\geq 3}(F) \geq 2$ or $n=6$, then by Lemma 4.2,

$$
\phi_{0}(F)+\phi_{1}(F)+\phi_{\text {dim }}(F) \leq 2 .
$$

Thus, if $\mathbf{0} \notin F$, then $\left|F^{1}\right|=n$ by the definition of potentials $\phi_{0}(F)$ and $\phi_{1}(F)$.

Now suppose that $\mathbf{0} \in F$. Consequently, $\left|F^{1}\right|=n-2$ by Lemma 4.3 and (6). Let $i \in[n]$ be such that $e_{i} \notin F^{1}$. Since $\phi_{\operatorname{dim}}(F)=0$ by $(6)$, we have $\left|F_{i: L}\right|=z(n-1)+1$. It follows that (3) holds for the set $F^{\prime}=F \backslash\{\mathbf{0}\}$. Hence, there exists a long $F^{\prime}$-free $u v$-path $P$ in $Q_{n}$ by Lemma 4.1. Moreover, since $\left|F_{i: L}^{1}\right|=n-2$, the path $P$ does not contain the vertex $\mathbf{0}$ by the second part of Lemma 4.1. Therefore, $P$ is also a long $F$-free $u v$-path, which is contrary to $(5)$.

In the next lemma we consider the configurations when $u$ or $v$ is $\mathbf{0}$ or there exists a dimension $i \in[n]$ such that $u, v \in V\left(Q_{i: R}\right)$.

Lemma 4.7. $u, v \neq \mathbf{0}$ and for every $i \in[n]$ it holds that $u_{i}=0$ or $v_{i}=0$. 
Proof. Without lost of generality, suppose for a contradiction that $u=\mathbf{0}$. Then $\phi_{0}(F)+$ $\phi_{1}(F) \geq n-1$ by the definition of potentials $\phi_{0}(F)$ and $\phi_{1}(F)$ since $\left|F^{1}\right|=|F(u)| \leq 3$, which contradicts Lemma 4.2. Thus, the first part holds.

For the second part, suppose that $u_{i}=v_{i}=1$ for some $i \in[n]$, so $u, v \in V\left(Q_{i: R}\right)$. Since $\left|F_{i: L}\right| \leq\left(\begin{array}{c}n-1 \\ 2\end{array}\right)-2$ by Corollary 4.5 , there is a long $F_{i: L}$-free cycle $C_{L}$ in $Q_{i: L}$ by induction (2). Let $a b$ be an edge of $C_{L}$ such that $a_{R}, b_{R} \notin F_{i: R}$ and $\left\{a_{R}, b_{R}\right\} \neq\{u, v\}$, and put $A=\left\{a_{R}, b_{R}\right\}, B=\{u, v\}$. Note that such edge $a b$ exists since $\left|C_{L}\right| \geq 2^{n-1}-2\left|F_{i: L}\right|$, every vertex of $F_{i: R} \cup\{u, v\}$ blocks at most 2 edges of $C_{L}$, and $2^{n-1}-2|F|-4 \geq 1$ for $n \geq 6$. Since

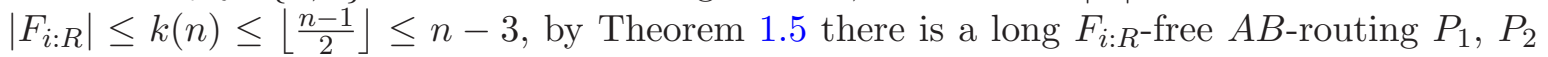
in $Q_{i: R}$. After interconnecting the path $C_{L}-\{a b\}$ and $P_{1}, P_{2}$ with the edges $a a_{R}, b b_{R}$ we obtain an $u v$-path in $Q_{n}-F$ of length

$$
\left|C_{L}\right|+\left|P_{1}\right|+\left|P_{2}\right|+1 \geq 2^{n-1}-2\left|F_{i: L}\right|+2^{n-1}-2\left|F_{i: R}\right|-3+1=2^{n}-2|F|-2,
$$

which contradicts with (5).

Next, we describe a construction based on long $F_{i: L}$-free cycles in $Q_{i: L}$. Without loss of generality, we assume that

$$
\begin{gathered}
\text { if }|u|=1 \text { or }|v|=1 \text {, then }|u|=1 ; \\
\text { if }|u|,|v| \geq 2 \text { and, }|u| \geq 3 \text { or }|v| \geq 3 \text {, then }|u| \geq 3 ; \\
\text { if }|u|=|v|=2 \text {, then }\left|F^{1} \cap N(u)\right| \geq\left|F^{1} \cap N(v)\right|
\end{gathered}
$$

otherwise, we switch the roles of $u$ and $v$. The last condition says that the vertex $u$ has at least the same number of faulty neighbors in the first level as the vertex $v$.

By Lemma 4.7, there exists a dimension $i \in[n]$ such that $u_{i}=0$ and $v_{i}=1$, so $u \in V\left(Q_{i: L}\right)$ and $v \in V\left(Q_{i: R}\right)$. Since $\left|F_{i: L}\right| \leq\left(\begin{array}{c}n-1 \\ 2\end{array}\right)-2$ by Corollary 4.5, there is an $F_{i: L}$-free cycle $C_{L}$ in $Q_{i: L}$ by induction (2). For the rest of this section, this splitting of $Q_{n}$ into $Q_{i: L}$ and $Q_{i: R}$, and the cycle $C_{L}$ are fixed. For ease of notation, we omit the index $i$ in the rest of this section.

For a vertex $z \in C_{L}$ let $c(z), a(z), z, b(z), d(z)$ be a subpath of $C_{L}$, and let $M(z)=$ $\{a(z), b(z), c(z), d(z)\}$. For example, see the set $M(u)$ on Figure $1(\mathrm{a})$. We say that a vertex $x$ of $Q_{L}$ is blocked if $x_{R} \in F_{R} \cup\{v\}$. Furthermore, we say that $M(z)$ is blocked if every vertex of $M(z)$ is blocked. The following proposition gives a sufficient condition which guarantees that the vertex $x$ cannot be blocked by the vertex $v$.

Proposition 4.8. For every vertex $x$ of $Q_{L}$, if $|x| \geq d(x, u)$, then $x_{R} \neq v$.

Proof. Recall that $i \in[n]$ is the fixed splitting dimension of $Q_{n}$ into $Q_{L}$ and $Q_{R}$, so $u_{i}=x_{i}=0$ and $v_{i}=1$. If $|x| \geq d(x, u)$, then there exists $j \in[n] \backslash\{i\}$ such that $u_{j}=x_{j}=1$ since $u \neq \mathbf{0}$ by Lemma 4.7. Furthermore, $v_{j}=0$ by Lemma 4.7. Hence $d(x, v) \geq 2$.

The next construction gives us many blocked vertices. For a vertex $x \in V\left(Q_{L}\right) \backslash F_{L}$ and the cycle $C_{L}$ let $\mathcal{S}(x)$ denote the following statement:

$$
\mathcal{S}(x):= \begin{cases}M(x) \text { is blocked } & \text { if } x \in C_{L}, \\ x \text { is blocked } & \text { if } x \notin C_{L} .\end{cases}
$$

Lemma 4.9. Let $C_{L}$ be a long $F_{L}$-free cycle in $Q_{L}, u \in V\left(Q_{L}\right)$, and $v \in V\left(Q_{R}\right)$. Then $\mathcal{S}(u)$ holds. Moreover, if $u \notin C_{L}$, then $\mathcal{S}(z)$ holds also for every neighbor $z$ of $u$ in $Q_{L}-F_{L}$. 


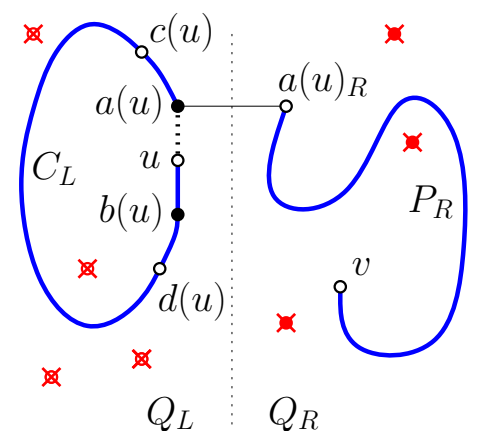

(a)

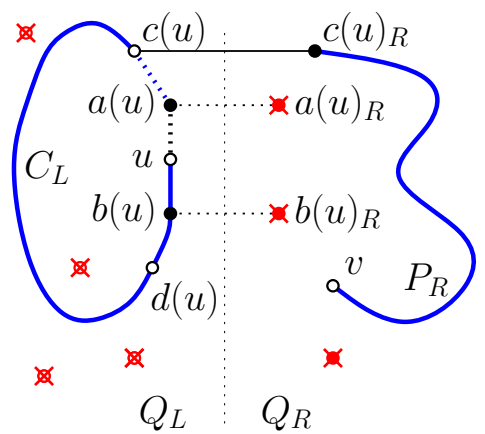

(b)

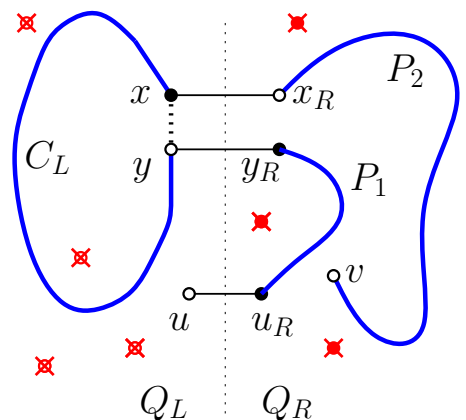

(c)

Figure 1: The construction in Lemma 4.9.

Proof. Case 1: $u \in C_{L}$. First, suppose that $a(u)$ or $b(u)$ is not blocked, say $a(u)_{R} \notin F_{R} \cup\{v\}$. See Figure 1(a) for an illustration. Then $Q_{R}$ contains a long $F_{R}$-free $a(u)_{R} v$-path $P_{R}$ by Corollary 1.3 since $\left|F_{R}\right| \leq k(n) \leq\left\lfloor\frac{n-1}{2}\right\rfloor$. By connecting $P_{R}$ and the path $C_{L}-\{u a(u)\}$ with the edge $a(u) a(u)_{R}$ we obtain an $u v$-path in $Q_{n}-F$ of length

$$
\left|C_{L}\right|+\left|P_{R}\right| \geq 2^{n-1}-2\left|F_{L}\right|+2^{n-1}-2\left|F_{R}\right|-2=2^{n}-2|F|-2,
$$

which is a contradiction with (5).

Second, suppose that $c(u)$ or $d(u)$ is not blocked, say $c(u)_{R} \notin F_{R} \cup\{v\}$. See Figure 1(b) for an illustration. Since $a(u), b(u)$ are blocked, it follows that $F_{R} \cup\left\{c(u)_{R}, v\right\}$ is not monopartite. Thus, by Corollary 1.6 there is an $c(u)_{R} v$-path $P_{R}$ in $Q_{R}-F_{R}$ of length at least $2^{n-1}-2\left|F_{R}\right|-1$. By connecting $P_{R}$ and the path $C_{L} \backslash\{u a(u), a(u) c(u)\}$ with the edge $c(u) c(u)_{R}$ we obtain an $u v$-path in $Q_{n}-F$ of length

$$
\left|C_{L}\right|+\left|P_{R}\right|-1 \geq 2^{n-1}-2\left|F_{L}\right|+2^{n-1}-2\left|F_{R}\right|-2=2^{n}-2|F|-2,
$$

which is a contradiction with (5).

Case 2: $u \notin C_{L}$. Next, suppose that the vertex $u$ is not blocked. Then, we choose an edge $x y$ on $C_{L}$ such that $x_{R}, y_{R} \notin F_{R}$. Note that such edge $x y$ exists since $\left|C_{L}\right| \geq 2^{n-1}-2\left|F_{L}\right|$, every vertex of $F_{R}$ blocks at most 2 edges of $C_{L}$, and $2^{n-1}-2|F| \geq 1$ for $n \geq 6$. See Figure $1(\mathrm{c})$ for an illustration. For sets $A=\left\{x_{R}, y_{R}\right\}, B=\left\{u_{R}, v\right\}$ we have that $A \neq B$ and $A \cup B$ is not monopartite. Hence, by Theorem 1.5 there is a long $F_{R}$-free $A B$-routing $P_{1}, P_{2}$ in $Q_{R}$. By connecting $u$, the path $C_{L}-\{x y\}$, and $P_{1}, P_{2}$ with the edges $x x_{R}, y y_{R}, u u_{R}$, we obtain an $u v$-path in $Q_{n}-F$ of length

$$
\left|C_{L}\right|+\left|P_{1}\right|+\left|P_{2}\right|+2 \geq 2^{n-1}-2\left|F_{L}\right|+2^{n-1}-2\left|F_{R}\right|-1=2^{n}-2|F|-1,
$$

which is contradiction with (5). Therefore, the statement $\mathcal{S}(u)$ is established.

Finally, suppose that $\mathcal{S}(z)$ does not hold for some neighbor $z \in V\left(Q_{L}\right) \backslash F_{L}$ of $u$. Then, by the same constructions as above, there is a long $F$-free $z v$-path $P$ in $Q_{n}$. Note that $u \notin P$. By prolonging $P$ with the edge $u z$ we obtain a long $F$-free $u v$-path in $Q_{n}$, contrary to (5).

In the next two lemmas we consider the configurations when the weight of the vertex $u$ or $v$ is not 2 . 
Lemma 4.10. $|u|,|v| \geq 2$.

Proof. Recall that $|u|,|v| \geq 1$ by Lemma 4.7. Suppose that $|u|=1$ or $|v|=1$, so $|u|=1$ by the assumption (7.1). It follows that $\left|F^{1}\right| \leq n-1$, so $n \geq 7$ by Lemma 4.6. First, we assume that $u \in C_{L}$. Then $M(u)$ is blocked by Lemma 4.9. Clearly, at least one of $a(u)$ and $b(u)$ has weight 2 , say $a(u)$, and $b(u)$ has weight 0 or 2 . If $|b(u)|=2$, then $a(u)_{R}, b(u)_{R} \in F_{R}$ by Proposition 4.8 and consequently, $\phi_{\geq 3}(F) \geq 2$ contrary to Lemma 4.6. Otherwise $|b(u)|=0$ and consequently, $\mathbf{0} \notin F,\left|F^{1}\right| \leq n-2$, and $\phi_{\geq 3}(F) \geq 1$ since $a(u)_{R} \in F_{R}$ by Proposition 4.8. Hence $\phi_{0}(F)+\phi_{1}(F)+\phi_{\geq 3}(F) \geq 5$, which contradicts Lemma 4.2.

Now, we have $u \notin C_{L}$. If $u$ has a neighbor $z$ on $C_{L}$ with $|z|=2$, then $M(z)$ is blocked by Lemma 4.9. Note that $a(z)$ or $b(z)$ belong to the third level, say $|a(z)|=3$, since $z$ has exactly two neighbors in the first level and one of them is $u \notin C_{L}$. Hence, we have $a(z)_{R} \in F_{R}$ by Proposition 4.8 and consequently, $\phi_{\geq 3}(F) \geq 2$, which contradicts Lemma 4.6.

Otherwise, no neighbor $z$ of $u$ in $Q_{L}-F_{L}$ with $|z|=2$ belongs to $C_{L}$. Since $|F(u)| \leq 3$, the vertex $u$ has at least $n-5$ neighbors $z$ in $Q_{L}-F_{L}$ with $|z|=2$. By Lemma 4.9, they are all blocked, but by Proposition 4.8, they are not blocked by the vertex $v$. Hence, $\phi_{\geq 3}(F) \geq n-5 \geq 2$ which contradicts Lemma 4.6.

Lemma 4.11. $|u|,|v| \leq 2$.

Proof. Suppose that $|u| \geq 3$ or $|v| \geq 3$, so $|u| \geq 3$ by the assumption (7.2). First, we consider the case when $u \in C_{L}$. Then $M(u)$ is blocked by Lemma 4.9. Since $a(u)$ and $b(u)$ belong to level at least 2 , we have $a(u)_{R}, b(u)_{R} \in F_{R}$ by Proposition 4.8 , so we obtain that $\phi_{\geq 3}(F) \geq 2$. Thus, $\left|F^{1}\right|=n$ by Lemma 4.6. Hence, the vertices $c(u)$ and $d(u)$ have weight at least 2 , and they are not blocked by the vertex $v$ by Proposition 4.8. Consequently $\phi_{\geq 3}(F) \geq 4$, which contradicts Corollary 4.4.

Now, we have $u \notin C_{L}$, so the vertex $u$ is blocked by Lemma 4.9. Since $u_{R} \in F_{R}$ by Proposition 4.8, we have $\phi_{\geq 3}(F) \geq 2$ and consequently, $\left|F^{1}\right|=n$ by Lemma 4.6. Furthermore, for an arbitrary neighbor $z \in V\left(Q_{L}\right) \backslash F_{L}$ of $u$ we obtain from Lemma 4.9 that $z$ is blocked if $z \notin C_{L}$, or $a(z)$ is blocked if $z \in C_{L}$. In both cases have another blocked vertex at distance at most 2 from $u$ and in level at least 2 , so $\phi_{\geq 3}(F) \geq 3$ by Proposition 4.8 , which contradicts Corollary 4.4.

By the previous two lemmas we have $|u|=|v|=2$. Let $u_{1}, u_{2}$ and $v_{1}, v_{2}$ be the neighbors of $u$ and $v$ of weight 1 , respectively. Note that from Lemma 4.7 it follows that these four vertices are distinct.

Lemma 4.12. $u_{1} \in F$ or $u_{2} \in F$.

Proof. Suppose that $u_{1}, u_{2} \notin F$. From the assumption (7.3) it follows that also $v_{1}, v_{2} \notin F$. Thus, $\phi_{1}(F) \geq 4$. If $u \in C_{L}$, then $M(u)$ is blocked by Lemma 4.9, and $c(u)$ or $d(u)$ is in level at least 2, say $|c(u)| \geq 2$, since they have the same parity as $u$. By Proposition 4.8 we have $c(u)_{R} \in F_{R}$ and consequently, $\phi_{\geq 3}(F) \geq 1$. Hence, we obtain that $\phi_{1}(F)+\phi_{\geq 3}(F) \geq 5$, a contradiction with Lemma 4.2.

If $u \notin C_{L}$, the vertex $u$ is blocked by Lemma 4.9. By Proposition 4.8 we have $u_{R} \in F_{R}$ and consequently, $\phi_{\geq 3}(F) \geq 1$. Similarly as above, we obtain that $\phi_{1}(F)+\phi_{\geq 3}(F) \geq 5$, a contradiction with Lemma 4.2 . 
The end of the proof of Theorem 1.4. If $u \in C_{L}$, then $M(u)$ is blocked by Lemma 4.9. From Lemma 4.12 it follows that $a(u)$ or $b(u)$ is in the third level, say $|a(u)|=3$. Furthermore, $|c(u)| \geq 2$. Since $a(u)_{R}, c(u)_{R} \in F_{R}$ by Proposition 4.8 , we have $\phi_{\geq 3}(F) \geq 3$, which contradicts Corollary 4.4 .

Finally, if $u \notin C_{L}$, then $u$ is blocked by Lemma 4.9. Let $z \in V\left(Q_{L}\right) \backslash F_{L}$ be an arbitrary neighbor of $u$ with $|z|=3$. Then by Lemma 4.9, $z$ is blocked, or the vertices $a(z)$ and $b(z)$ of weight at least 2 are blocked. By Proposition 4.8, $u_{R}, z_{R} \in F_{R}$ in the first case, and $u_{R}, a(z)_{R}, b(z)_{R} \in F_{R}$ in the latter case. Altogether, we obtain that $\phi_{\geq 3}(F) \geq 3$, which is a final contradiction with Corollary 4.4.

Therefore, we conclude that the contradicted assumption (5) is false, i.e. the statement of Theorem 1.4 holds.

\section{$5 \quad$ Long cycles}

In this section we prove the main Theorem 1.1 which says that for every set of faulty vertices $F$ of $Q_{n}$ of size at most $\left(\begin{array}{c}n \\ 2\end{array}\right)-2$ there exists a cycle in $Q_{n}-F$ of length at least $2^{n}-2|F|$, where $n \geq 4$. Such cycle is called a long $F$-free cycle.

$\mathrm{Fu}$ [7] proved that there exists a long $F$-free cycle if $|F| \leq 2 n-4$, where $n \geq 3$, which implies that Theorem 1.1 holds for $n=4$. Theorem 3.1 implies the base of induction of Theorem 1.1 for $n=5$.

In the induction step of the proof of Theorem 1.1 for $n$, we assume that both Theorems 1.1 and 1.4 hold for $n-1$; see (2). Let us consider a fixed set $F$ of at most $\left(\begin{array}{l}n \\ 2\end{array}\right)-2$ faulty vertices in $Q_{n}$, where $n \geq 6$. Furthermore, we assume that $\left|F_{i: L}\right| \geq\left|F_{i: R}\right|$ for every dimension $i \in[n]$; see (1).

\subsection{Induction-friendly split}

In the first part of the proof of Theorem 1.1 we assume that there exists a dimension $i \in[n]$ such that $\left|F_{i: L}\right|,\left|F_{i: R}\right| \leq\left(\begin{array}{c}n-1 \\ 2\end{array}\right)-2$; see (3). In this case we apply induction (2) in both $Q_{i: L}$ and $Q_{i: R}$ to construct a long $F$-free cycle $Q_{n}$. Moreover, the following lemma also considers other conditions in which we can simply find a long $F$-free cycle in the same way. Those conditions are useful later.

Lemma 5.1. If there exists a dimension $i \in[n]$ such that at least one of the following conditions holds, then there exists a long $F$-free cycle in $Q_{n}$.

(i) There exists a long $F_{i: L}$-free cycle $C_{L}$ in $Q_{i: L}$;

(ii) $\left|F_{i: L}\right| \leq\left(\begin{array}{c}n-1 \\ 2\end{array}\right)-2$;

(iii) $\left|F_{i: L}\right|=\left(\begin{array}{c}n-1 \\ 2\end{array}\right)-1$ and there exists $x \in F_{i: L}$ having at most one $F_{i: L}$-free neighbor in $Q_{i: L}$.

Proof. Our first aim is to find a long $F_{i: L}$-free cycle $C_{L}$ in $Q_{i: L}$. If the condition (i) is satisfied, then the cycle is given. If the condition (ii) is satisfied, then the cycle exists by induction (2).

Let us assume that the condition (iii) is satisfied. Let $F^{\prime}=F_{i: L} \backslash\{x\}$. By induction (2), there exists a long $F^{\prime}$-free cycle $C_{L}$ in $Q_{i: L}$. Since no cycle of $Q_{i: L}-F^{\prime}$ contains $x$, the cycle $C_{L}$ is also $F_{i: L}$-free. 
Our next aim is to find an edge $x y$ of $C_{L}$ such that

$$
x_{R}, y_{R} \notin F_{i: R} \text { and }\left|F_{i: R}\left(x_{R}\right)\right|,\left|F_{i: R}\left(y_{R}\right)\right| \leq 3 .
$$

If there exists an edge $x y$ satisfying (8), then by induction (2), there is a long $F_{i: R}$-free $x_{R} y_{R}$-path $P_{R}$ in $Q_{i: R}$ since $\left|F_{i: R}\right| \leq\left\lfloor\frac{|F|}{2}\right\rfloor \leq\left\lfloor\frac{(n-1)^{2}+(n-1)-4}{4}\right\rfloor$. We replace the edge $x y$ in $C_{L}$ by a path $\left(x, x_{R}, P_{R}, y_{R}, y\right)$ and we obtain an $F$-free cycle in $Q_{n}$ of length at least

$$
\left(2^{n-1}-2\left|F_{i: L}\right|-1\right)+2+\left(2^{n-1}-2\left|F_{i: R}\right|-1\right)=2^{n}-2|F| .
$$

It remains to show that there exists an edge $x y$ satisfying (8). Recall that $\alpha\left(F_{i: R}\right)$ is the number of vertices $z$ in $Q_{i: R}$ with $\left|F_{i: R}(z)\right| \geq 4$. There are at most $\left|F_{i: R}\right|+\alpha\left(F_{i: R}\right)$ vertices that cannot be used as end-vertices of a long $F_{i: R}$-free path in $Q_{i: R}$. Since the length of $C_{L}$ is at least $2^{n-1}-2\left|F_{i: L}\right|$, the number of edges $x y$ satisfying (8) is at least

$$
2^{n-1}-2\left|F_{i: L}\right|-2\left(\left|F_{i: R}\right|+\alpha\left(F_{i: R}\right)\right) \geq 2^{n-1}-2|F|-2 \alpha\left(F_{i: R}\right) \geq 1 .
$$

The last inequality follows from $\left|F_{i: R}\right| \leq|F| / 2$ and from

- Proposition 2.2 for $n=6$;

- the inequality $\alpha\left(F_{i: R}\right) \leq\left(\begin{array}{c}\left|F_{i: R}\right| \\ 2\end{array}\right) / 3$ by Proposition 2.1 for $n=7$;

- the inequality $\alpha\left(F_{i: R}\right) \leq \frac{(n-1)\left|F_{i: R}\right|}{4}$ by Proposition 2.1 for $n \geq 8$.

\subsection{Potentials}

In the second part of the proof of Theorem 1.1 we assume that (3) fails, i.e. (4) holds.

Let us recall that we use the following potentials, where now we have $z(n)=\left(\begin{array}{l}n \\ 2\end{array}\right)-2$.

$$
\begin{array}{rlrl}
\phi(F)=\phi_{0}(F)+\phi_{1}(F)+\phi_{\geq 3}(F)+\phi_{\operatorname{dim}}(F), & \\
\phi_{1}(F) & =n-\left|F^{1}\right|, \\
\phi_{0}(F) & =2-2\left|F^{0}\right|, & \phi_{\operatorname{dim}}(F) & =\sum_{i \in[n]}\left(\left|F_{i: L}\right|-z(n-1)-1\right) .
\end{array}
$$

By substituting $z(n)=\left(\begin{array}{l}n \\ 2\end{array}\right)-2$ and $k(n)=z(n)-z(n-1)-1=n-2$ into Proposition 3.2 , the next lemma follows immediately.

Lemma 5.2. Let $F$ be a set of faulty vertices of $Q_{n}$ of size at most $\left(\begin{array}{c}n \\ 2\end{array}\right)-2$. If $\left|F_{i: L}\right| \geq\left(\begin{array}{c}n-1 \\ 2\end{array}\right)-1$ for every dimension $i \in[n]$, then $|F| \geq\left(\begin{array}{l}n \\ 2\end{array}\right)-3$ and

$$
\phi(F)= \begin{cases}6 & \text { if }|F|=\left(\begin{array}{l}
n \\
2
\end{array}\right)-2 \\
8-n & \text { if }|F|=\left(\begin{array}{l}
n \\
2
\end{array}\right)-3 .\end{cases}
$$

In the rest of this section we proceed by contradiction. Therefore, we consider a set of vertices $F$ of $Q_{n}$ of size at most $\left(\begin{array}{l}n \\ 2\end{array}\right)-2$ such that

$$
\text { there is no long } F \text {-free cycle in } Q_{n} \text {. }
$$


From the assumption (ii) of Lemma 5.1 it follows that $\left|F_{i: L}\right| \geq\left(\begin{array}{c}n-1 \\ 2\end{array}\right)-1$ and $\left|F_{i: R}\right| \leq n-2$ for every dimension $i \in[n]$; see (4).

It follows from Lemma 5.2 that there cannot be too many vertices in $F^{\geq 3}$ and they cannot be too far from $\mathbf{0}$. Now, we present a construction which gets a faulty vertex $a$ and gives us another faulty vertex $b_{R}$ in the level $|a|$ or $|a|+2$.

Lemma 5.3. Let $i \in[n]$ be a dimenstion and let a be a given vertex of $F_{i: L}^{k}$. Let one of the two following conditions hold.

(i) $\left|F_{i: L}\right|=\left(\begin{array}{c}n-1 \\ 2\end{array}\right)-1$,

(ii) $\left|F_{i: L}\right|=\left(\begin{array}{c}n-1 \\ 2\end{array}\right),\left|F_{i: L}^{1} \backslash\{a\}\right| \geq n-2, \mathbf{0} \in F$ and $a \neq \mathbf{0}$.

Then, there exists $b \in V\left(Q_{i: L}\right) \cap N(a)$ such that $b_{R} \in F_{i: R}$. Hence, $\left|b_{R}\right| \in\{k, k+2\}$.

Moreover, if at least one of the three following conditions holds, then $\left|b_{R}\right|=k+2$.

(iii) Every vertex $x \in N^{-}(a)$ is faulty,

(iv) for every $x \in N^{-}(a)$ the vertex $x_{R}$ is $F_{i: R}$-free,

(v) $\left|F_{i: L}^{1}\right|=n-1$ and $k=1$.

Proof. Let

$$
F^{\prime}= \begin{cases}F_{i: L} \backslash\{a\} & \text { if (i) holds, } \\ F_{i: L} \backslash\{a, \mathbf{0}\} & \text { if (ii) holds. }\end{cases}
$$

By induction (2), there exists a long $F^{\prime}$-free cycle $C_{L}$ in $Q_{i: L}$. If (ii) holds, then $\mathbf{0} \notin C_{L}$ because $\mathbf{0}$ has at most one $F^{\prime}$-free neighbor in $Q_{i: L}$. Since there is no long $F_{i: L}$-free cycle in $Q_{i: L}$ by the assumption (i) of Lemma 5.1 and by the contradicted assumption (9), the vertex $a$ is contained in $C_{L}$.

Let $b$ and $c$ be two neighbors of $a$ on $C_{L}$. If $b_{R}, c_{R} \notin F_{i: R}$, then by Theorem 1.2 there exists a long $F_{i: R}$-free $b_{R} c_{R}$-path $P_{R}$ in $Q_{i: R}$ since $\left|F_{i: R}\right| \leq n-2$ and $b_{R}, c_{R}$ are not adjacent. Hence, the length of an $F$-free cycle obtained from $C_{L}$ by removing edges $b a, a c$ and inserting a path $\left(b, b_{R}, P_{R}, c_{R}, c\right)$ is at least

$$
\left(2^{n-1}-2\left|F^{\prime}\right|\right)-2+2+\left(2^{n-1}-2\left|F_{i: R}\right|-2\right) \geq 2^{n}-2|F| .
$$

Therefore, at least one of $b_{R}$ and $c_{R}$ belongs into $F_{i: R}$, say $b_{R} \in F_{i: R}$, which implies the first part of the statement.

Now, we prove the second part. Note that

$$
\left|b_{R}\right|= \begin{cases}k & \text { if } b \in N^{-}(a), \\ k+2 & \text { if } b \in N^{+}(a) .\end{cases}
$$

If $b \in N^{-}(a)$, then neither the condition (iii) nor (iv) is satisfied since $b \notin F_{i: L}$ and $b_{R} \in F_{i: R}$. If (v) holds, then $b \in N^{+}(a)$; otherwise, the vertex $a$ is the only $F^{\prime}$-free neighbor of $b=\mathbf{0}$ in $Q_{i: L}$, and there is no cycle in $Q_{i: L}-F^{\prime}$ containing $\mathbf{0}$, but $b \in C_{L}$. 
This lemma is useful to find a faulty vertex in $F^{\geq 3}$ which increases the potential $\phi_{\geq 3}(F)$. We often combine this lemma with other observations to show that the potential $\phi(F)$ is greater than the value given by Lemma 5.2 which provides us with a contradiction. One such example follows, compare it with Lemma 4.3 in the previous section.

For practical purposes, we say that we use Lemma 5.3 with the assumption (i) on a vertex $x \in F_{i: L}$ to obtain a vertex $y \in V\left(Q_{i: L}\right)$. This only means that $Q_{n}$ is split by the dimension $i$, and we apply Lemma 5.3 for the given vertex $a=x$ such that the assumption (i) is satisfied. Then, $y$ is the vertex $b$ obtained by Lemma 5.3. Similarly, we say that we use Lemma 5.3 with the assumption (ii) and (iii) on a vertex $x \in F_{i: L}$ to obtain a vertex $z \in F_{i: R}$. This only means that the dimension $i$ and the vertex $a=x$ satisfy both conditions (ii) and (iii) and $z$ is the vertex $b_{R} \in F_{i: R}$ in level $|a|+2$ obtained by Lemma 5.3. Note that $d(x, z)=2$.

Lemma 5.4. $0 \notin F$ or $\left|F^{1}\right| \leq n-2$.

Proof. For a contradiction, let us suppose that $\mathbf{0} \in F$ and $\left|F^{1}\right| \geq n-1$.

If there exists a dimension $i$ such that $\left|F_{i: L}\right|=\left(\begin{array}{c}n-1 \\ 2\end{array}\right)-1$, then by Lemma 5.1 with the assumption (iii) for $x=\mathbf{0} \in F$, which has at most one $F_{i: L}$-free neighbor in $Q_{i: L}$, we obtain a long $F$-free cycle in $Q_{n}$ which is a contradiction with (9).

Now, we assume that there is no dimension $i \in[n]$ such that $\left|F_{i: L}\right|=\left(\begin{array}{c}n-1 \\ 2\end{array}\right)-1$, so $\phi_{\operatorname{dim}}(F) \geq n$. This is possible, by Lemma 5.2, only if $\phi_{\operatorname{dim}}(F)=n=6=\phi(F)$ and hence by the definition of $\phi(F)$ we have that $\left|F^{1}\right|=6,0 \in F$ and $F^{\geq 3}=\emptyset$. Note that in this case $\left|F_{i: L}\right|=\left(\begin{array}{c}n-1 \\ 2\end{array}\right)$ for every dimension $i \in[n]$, so we use Lemma 5.3 with the assumptions (ii) and (iii) on some vertex $a \in F_{i: L}^{1}$ to obtain a vertex in $F_{i: R}^{3}$, which is a contradiction with $F^{\geq 3}=\emptyset$.

Lemma 5.4 implies that $\phi_{0}(F)+\phi_{1}(F) \geq 2$. Hence, $\phi_{\geq 3}(F)+\phi_{d i m}(F) \leq 4$ by Lemma 5.2 which implies that

$$
\text { there exists a dimension } i \in[n] \text { such that }\left|F_{i: L}\right|=\left(\begin{array}{c}
n-1 \\
2
\end{array}\right)-1 \text {, }
$$

since $n \geq 6$. Moreover, the definition of $\phi_{\operatorname{dim}}(F)$ implies for a given vertex $x$ of $Q_{n}$ that

$$
\text { if } \phi_{\operatorname{dim}}(F)+|x|<n \text {, then } \exists i \in[n] \text { such that }\left|F_{i: L}\right|=\left(\begin{array}{c}
n-1 \\
2
\end{array}\right)-1 \text { and } x \in V\left(Q_{i: L}\right) \text {, }
$$

because at least $n-\phi_{\text {dim }}(F)$ dimensions $i \in[n]$ satisfy $\left|F_{i: L}\right|=\left(\begin{array}{c}n-1 \\ 2\end{array}\right)-1$, and at most $|x|$ of those dimensions volatile $x \in V\left(Q_{i: L}\right)$.

Our proof still proceeds by contradiction (9). In the following two lemmas we prove that $\phi_{0}(F)+\phi_{1}(F) \geq 3$. In the first one we consider the case when $\mathbf{0} \notin F$ and $\left|F^{1}\right|=n$; and in the second one, the case when $\mathbf{0} \in F$ and $\left|F^{1}\right|=n-2$.

Lemma 5.5. $\left|F^{1}\right| \leq n-1$.

Proof. For a contradiction we suppose that $\left|F^{1}\right|=n$. Hence, $\mathbf{0} \notin F$ by Lemma 5.4. We proceeds in three steps. First, we prove that $\left|F^{3}\right| \geq 1$. Next, we prove that $\left|F^{4}\right| \geq 1$, which we finally improve to $\left|F^{4}\right| \geq 2$. This is a contradiction to Lemma 5.2.

By (10) we split $Q_{n}$ by such dimension $i \in[n]$ that $\left|F_{i: L}\right|=\left(\begin{array}{c}n-1 \\ 2\end{array}\right)-1$. We use Lemma 5.3 with the assumptions (i) and (v) on some vertex of $F_{i: L}^{1}$ to obtain $\left|F_{i: R}^{3}\right| \geq 1$. By Lemma 5.2 we know that $|F|=\left(\begin{array}{c}n \\ 2\end{array}\right)-2$ since $\phi_{0}(F)+\phi_{\geq 3}(F) \geq 3$ and $n \geq 6$. 
We observe that $F_{i: L}^{2} \neq \emptyset$; otherwise $\left|F_{i: L}^{\geq 3}\right|=\left|F_{i: L}\right|-\left|F_{i: L}^{1}\right|=\left(\begin{array}{c}n-1 \\ 2\end{array}\right)-1-(n-1) \geq 4$ which implies $\phi_{\geq 3}(F) \geq\left|F_{i: L}^{\geq 3}\right|+\left|F_{i: R}^{3}\right| \geq 5$, contrary to Lemma 5.2. Hence, we use Lemma 5.3 with the assumptions (i) and (iii) on some vertex of $F_{i: L}^{2}$ to obtain a vertex $x \in F_{i: R}^{4}$.

Now, we know that $F^{3}, F^{4} \neq \emptyset$ and $0 \notin F$ which implies $\phi_{\operatorname{dim}}(F) \leq 1$ by Lemma 5.2. Therefore, there exists a dimension $j$ such that $\left|F_{j: L}\right|=\left(\begin{array}{c}n-1 \\ 2\end{array}\right)-1$ and $x \in F_{j: L}^{4}$ by (11). We use Lemma 5.3 with the assumption (i) on the vertex $x$ to obtain a vertex in $F_{j: R}^{\geq 4}$. Hence, $\left|F^{\geq 4}\right| \geq 2$ and $\left|F^{3}\right| \geq 1$, so $\phi_{\geq 3}(F) \geq 5$. It implies $\phi(F) \geq \phi_{0}(F)+\phi_{\geq 3}(F) \geq 7$, which is a contradiction with Lemma 5.2.

Lemma 5.6. If $\mathbf{0} \in F$, then $\left|F^{1}\right| \leq n-3$.

Proof. For a contradiction we suppose that $\mathbf{0} \in F$ and $\left|F^{1}\right|=n-2$. First, we prove that $\phi_{\operatorname{dim}}(F) \geq 2$. Next, we prove that there exist two vertices $x$ and $y$ in $F_{d: R}^{3}$ for some $d \in[n]$. Finally, we show that there exist 4 distinct dimensions $d_{1}, d_{2}, d_{3}, d_{4} \in[n]$, satisfying $x \in F_{d_{l}: R}^{3}$ for $l \in[4]$ which implies that $|x| \geq 4$, contrary to $x \in F^{3}$.

Let $e_{i}$ and $e_{j}$ be the (only) two $F$-free vertices in the first level. We observe that $\left|F_{i: L}\right|,\left|F_{j: L}\right| \geq\left(\begin{array}{c}n-1 \\ 2\end{array}\right)$; otherwise we use Lemma 5.1 with the assumption (iii) on the vertex 0 to obtain a contradiction with (9). Therefore, $\phi_{1}(F)+\phi_{\operatorname{dim}}(F) \geq 4$; and consequently, $\phi_{\geq 3}(F) \leq 2$ by Lemma 5.2.

We split $Q_{n}$ by a dimension $d \in[n]$ so that $\left|F_{d: L}\right|=\left(\begin{array}{c}n-1 \\ 2\end{array}\right)-1$ by $(10)$. Let $a^{1}, a^{2}, a^{3}$ be arbitrary distinct vertices of $F_{d: L}^{1}$. Note that such vertices exist since $\left|F_{d: L}^{1}\right|=n-3$ and $n \geq 6$. We use Lemma 5.3 with the assumptions (i) and (iii) for every vertex $a^{m}$ to obtain $b^{m} \in$ $V\left(Q_{d: L}\right)$ such that $b_{R}^{m} \in F_{d: R}^{3}$, where $m \in[3]$. Note that $\left|\left\{b_{R}^{1}, b_{R}^{2}, b_{R}^{3}\right\}\right| \leq 2$ since $\phi_{\geq 3}(F) \leq 2$. On the other hand, if $b_{R}^{1}=b_{R}^{2}=b_{R}^{3}$, then $b^{1}=b^{2}=b^{3}$; so $b^{1} \in N^{+}\left(a^{1}\right) \cap N^{+}\left(a^{2}\right) \cap N^{+}\left(a^{3}\right)$, but $\left|N^{-}\left(b^{1}\right)\right|=2$. Hence, $b_{R}^{1}, b_{R}^{2}$ and $b_{R}^{3}$ are two different vertices; say $x_{d}$ and $y_{d}$. Furthermore, $F^{3} \mid=\phi_{\geq 3}(F)=\phi_{\text {dim }}(F)=2$.

Since $\phi_{\operatorname{dim}}(F)=2$ and $n \geq 6$, there are at least 4 distinct dimensions $d_{l} \in[n], l \in[4]$, such that $\left|F_{d_{l}: L}\right|=\left(\begin{array}{c}n-1 \\ 2\end{array}\right)-1$. For every dimension $d_{l}$ we obtain vertices $x_{d_{l}}, y_{d_{l}} \in F_{d_{l}: R}^{3}$ in the same way as described in the previous paragraph. Since $\left|F^{3}\right|=2$, the pairs of vertices $x_{d_{l}}$ and $y_{d_{l}}$ are the same for all $l \in[4]$; say $x_{d_{1}}=x_{d_{2}}=x_{d_{3}}=x_{d_{4}}$. But $x_{d_{1}} \in F_{d_{l}: R}^{3}$ for all $l \in[4]$ implies $\left|x_{d_{1}}\right| \geq 4$ which contradicts $x_{d_{1}} \in F^{3}$.

Note that from Lemmas 5.5 and 5.6 it follows that $\phi_{1}(F)+\phi_{0}(F) \geq 3$. Therefore, Lemma 5.2 implies the following statement since $n \geq 6$.

Corollary 5.7. $\phi_{\geq 3}(F)+\phi_{\text {dim }}(F) \leq 3$ and $|F|=\left(\begin{array}{l}n \\ 2\end{array}\right)-2$.

Consequently, from (11) we obtain that for every vertex $a \in F$

$$
\text { there exists a dimension } i \in[n] \text { such that } a \in F_{i: L} \text { and }\left|F_{i: L}\right|=\left(\begin{array}{c}
n-1 \\
2
\end{array}\right)-1 \text {. }
$$

Let $u \vee v$ denote the vertex $w=\left(w_{1}, w_{2}, \ldots, w_{n}\right)$ with $w_{i}=u_{i} \vee v_{i}$ for all $i \in[n]$, where $\vee$ is the logical disjunction. Note that $w \in Q_{i: L}$ if and only if $u, v \in Q_{i: L}$ for every dimension $i \in[n]$.

Lemma 5.8. $F^{\geq 3}=\emptyset$. 
Proof. For a contradiction, let us suppose that there exists a vertex $a \in F^{\geq 3}$. We proceed in 4 steps. First, we prove that $F^{\geq 4}=\emptyset$. Each of next three steps splits $Q_{n}$ and uses Lemma 5.3 to obtain a new vertex in $F^{3}$, which implies that $\left|F^{3}\right| \geq 4$, contrary to Corollary 5.7. Note that those three splits use different dimensions.

If $|a| \geq 4$, then we split $Q_{n}$ so that $a \in F_{i: L}$ and $\left|F_{i: L}\right|=\left(\begin{array}{c}n-1 \\ 2\end{array}\right)-1$ by (12). Then we use Lemma 5.3 with the assumption (i) on the vertex $a$ to obtain another faulty vertex in level at least 4 , which is a contradiction with Corollary 5.7. Therefore, we assume that $F^{\geq 4}=\emptyset$ and $a \in F^{3}$.

We split $Q_{n}$ so that $a \in V\left(Q_{i: L}\right)$ and $\left|F_{i: L}\right|=\left(\begin{array}{c}n-1 \\ 2\end{array}\right)-1$ by (12). By Lemma 5.3 with the assumption (i), there exists $b \in F_{i: R}$ such that $|b|=3$ and $d(a, b)=2$. Hence, $\phi_{\geq 3}(F) \geq 2$.

Let $x=a \vee b$. Since $d(a, b)=2$ and $|a|=|b|=3$, we have $|x|=4$. Since $\phi_{\text {dim }}(F) \leq 1$ by Corollary 5.7, there exists a dimension $j$ such that $x \in V\left(Q_{j: L}\right)$ and $\left|F_{j: L}\right|=\left(\begin{array}{c}n-1 \\ 2\end{array}\right)-1$ by (11). Hence $a, b \in F_{j: L}$. We use Lemma 5.3 with the assumption (i) twice on both $a$ and $b$ to obtain $c, d \in F_{j: R}^{3}$ such $d(a, c)=d(b, d)=2$. Since $\phi_{\geq 3}(F) \leq 3$ by Corollary 5.7 , we have $c=d$. Hence, $|a|=|b|=|c|=3$ and $d(a, b)=d(a, c)=\bar{d}(b, c)=2$.

Let $y=a \vee b \vee c$. Similarly, we have $|y| \leq 5$ and $\phi_{\operatorname{dim}}(F)=0$, so there exists a dimension $d$ such that $y \in V\left(Q_{d: L}\right)$ and $\left|F_{d: L}\right|=\left(\begin{array}{c}n-1 \\ 2\end{array}\right)-1$ by (11). Using Lemma 5.3 with the assumption (i) on the vertex $a$ we obtain a faulty vertex in $F_{d: R}^{3}$; so $\left|F^{3}\right| \geq 4$, which is a contradiction with Corollary 5.7.

Lemma 5.9. $0 \notin F$.

Proof. For a contradiction we suppose that $\mathbf{0} \in F$. Hence, $\left|F^{1}\right| \leq n-3$ by Lemma 5.6.

We observe that $F^{1}=\emptyset$, otherwise we choose $x \in F^{1}$, we split $Q_{n}$ so that $x \in F_{i: L}$ and $\left|F_{i: L}\right|=\left(\begin{array}{c}n-1 \\ 2\end{array}\right)-1$ by (12), and by Lemma 5.3 with the assumptions (i) and (iii) we obtain $F^{\geq 3} \neq \emptyset$, contrary to Lemma 5.8. Hence, $\phi_{1}(F)=n$ which is possible only if $n=6,\left|F^{1}\right|=0$, $\left|F^{2}\right|=12$ and $\phi_{\operatorname{dim}}(F)=0$.

Since $\phi_{\operatorname{dim}}(F)=0$, we have $\left|F_{i: R}\right|=k(n)=n-2=4$ for every dimension $i \in[n]$. Since $\left|F^{1}\right|,\left|F^{\geq 3}\right|=\emptyset$, only one vertex of $N^{+}\left(e_{i}\right)$ is $F$-free for every vertex $e_{i}$ of the first level in $Q_{n}$. Therefore, for every dimension $j$ there exists exactly one other dimension $k$ such that $e_{j, k} \notin F$, so all dimensions are split into three pairs $\left\{j_{1}, k_{1}\right\},\left\{j_{2}, k_{2}\right\}$ and $\left\{j_{3}, k_{3}\right\}$ such that $e_{j_{1}, k_{1}}, e_{j_{2}, k_{2}}, e_{j_{3}, k_{3}} \notin F$. This is satisfied up to isomorphism only by one set of faulty vertices $F$ : the set of all vertices of level 0 or 2 except the vertices $e_{1,2}, e_{3,4}$ and $e_{5,6}$. By Lemma 5.1 with the assumption (i), it suffices to find a long $F_{6: L}$-free cycle in $Q_{6: L}$ which is presented on Figure 2. Thus, we obtain a contradiction with (9).

Finally, we prove the last simple lemma which leads to a contradiction with (9).

Lemma 5.10. For every dimension $i \in[n]$, if $e_{i} \notin F$, then $\left|F_{i: L}\right| \geq\left(\begin{array}{c}n-1 \\ 2\end{array}\right)$.

Proof. Let us consider a vertex $e_{i} \notin F$ such that $\left|F_{i: L}\right|=\left(\begin{array}{c}n-1 \\ 2\end{array}\right)-1$. There exists a vertex $x \in F_{i: L}^{1}$, because $\phi_{1}(F) \leq 4 \leq n-2$ by Lemmas 5.2 and 5.9. We use Lemma 5.3 with the assumptions (i) and (iv) on the vertex $x$ to obtain $\left|F^{3}\right| \geq 1$, which is a contradiction to Lemma 5.8.

The end of the proof of Theorem 1.1. Recall that $\phi_{0}(F)=2$ by Lemma 5.9, which implies that $\phi_{\operatorname{dim}}(F)+\phi_{1}(F) \leq 4$ by Lemma 5.2. Lemma 5.10 says that $\phi_{\operatorname{dim}}(F) \geq \phi_{1}(F)$ which implies that $\left|F^{1}\right| \geq n-2$. On the other hand, we know that $\left|F^{1}\right| \leq n-1$ by Lemma 5.5. Moreover, $|F|=\left(\begin{array}{c}n \\ 2\end{array}\right)-2$ and every faulty vertex is in the level 1 or 2 by Lemmas 5.8 and 5.9. 


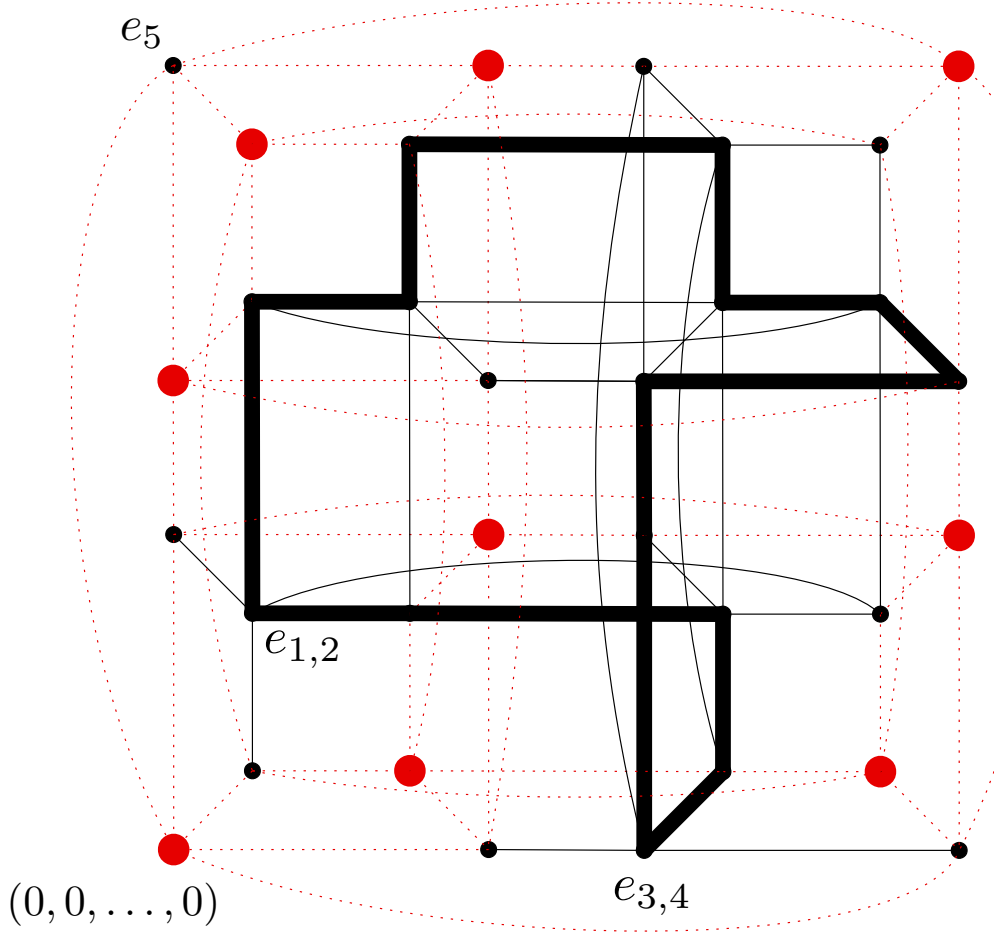

Figure 2: Bold points are faulty vertices and bold lines form a long $F_{6: L}$-free cycle in $Q_{6: L}$ for Lemma 5.9.

If there exists a vertex $a \in F^{2}$ such that both vertices in $N^{-}(a)$ are faulty, then we split $Q_{n}$ so that $a \in F_{i: L}$ and $\left|F_{i: L}\right|=\left(\begin{array}{c}n-1 \\ 2\end{array}\right)-1$ by (12). Then, we use Lemma 5.3 with the assumptions (i) and (iii) to obtain $\left|F^{4}\right| \geq 1$, which is a contradiction to Lemma 5.8. Hence, every vertex of $F^{2}$ is above some $F$-free vertex of level 1 .

Lemma 5.10 also implies that there are at most $n-3$ faulty vertices above every $F$-free vertex in level 1. Since there are at most two $F$-free vertices in level 1, we have $\left|F^{2}\right| \leq 2(n-3)$. This leads to the final contradiction $3 n-7 \geq\left|F^{1}\right|+\left|F^{2}\right|=|F|=\left(\begin{array}{l}n \\ 2\end{array}\right)-2$ since $n \geq 6$, which finishes the proof of the main Theorem 1.1.

Acknowledgement. We would like to Tomáš Dvořák for fruitful discussion and Václav Koubek also for many comments which improved the presentation of this paper.

\section{References}

[1] N. Castañeda and I.S. Gotchev. Embedded paths and cycles in faulty hypercubes. Journal of Combinatorial Optimization, 2009. DOI: 10.1007.s10878-008-9205-6.

[2] M.Y. Chan and S.-J. Lee. Distributed fault-tolerant embedding of rings in hypercubes. Journal of Parallel and Distributed Computing, 11:63-71, 1991.

[3] T. Dvořák and V. Koubek. Long paths in hypercubes with a quadratic number of faults. Information Sciences, 179:3763-3771, 2009. 
[4] T. Dvořák and V. Koubek. On the complexity of finding long paths and cycles in faulty hypercubes. Submitted., 2009.

[5] J. Fink and P. Gregor. Long pairs of paths in faulty hypercubes. Technical Report 465, Institute for Theoretical Computer Science, Charles University, Malostransé náměstí 25, 11800 Praha 2, Czech Republic, 2009.

[6] J. Fink and P. Gregor. Long paths and cycles in faulty hypercubes. Information Sciences, 179:3634-3644, 2009.

[7] J.-S. Fu. Fault-tolerant cycle embedding in the hypercube. Parallel Computing, 29(6):821-832, 2003.

[8] J.-S. Fu. Longest fault-free paths in hypercubes with vertex faults. Information Sciences, 176(7):759-771, 2006.

[9] C.-N. Hung, Y.-H. Chang, and C.-M. Sun. Longest paths and cycles in faulty hypercubes. In Parallel and Distributed Computing and Networks, pages 101-110, 2006.

[10] V. Koubek, 2008. Private communication.

[11] T.-L. Kueng, T. Liang, L.-H. Hsu, and J.J.M Tan. Long paths in hypercubes with conditional node-faults. Information Sciences, 179:667-681, 2009.

[12] M. Lewinter and W. Widulski. Hyper-hamilton laceable and caterpillar-spannable product graphs. Computers $\&$ Mathematics with Applications, 34(11):99-104, 1997.

[13] Y.-C. Tseng. Embedding a ring in a hypercube with both faulty links and faulty nodes. Information Processing Letters, 59(4):217-222, 1996.

[14] J.-M. Xu and M. Ma. Survey on path and cycle embedding in some networks. Frontiers of Mathematics in China, 4:217-252, 2009.

[15] P.J. Yang, S.B. Tien, and C.S. Raghavendra. Embedding of rings and meshes onto faulty hypercubes using free dimensions. IEEE Transactions on Computers, 43:608-613, 1994. 


\section{Appendix: Long routings with two paths}

In this appendix we include the proofs of Theorem 1.5 and Corollary 1.6 from the paper [5]. The authors would like to study similar problems more intensively and publish them separately, but Theorem 1.5 and Corollary 1.6 are used in this paper, so their proofs are included for the purpose of referee.

Recall that for a set $F \subseteq V\left(Q_{n}\right)$ and two different sets $A, B \subseteq V\left(Q_{n}\right) \backslash F$ with $|A|=|B|=$ 2, a pair $P_{1}, P_{2}$ of vertex-disjoint $A B$-paths in $Q_{n}-F$ is called a long $F$-free $A B$-routing if $\left|P_{1}\right|+\left|P_{2}\right| \geq 2^{n}-2|F|-3$. Note that if $P_{1}$ and $P_{2}$ have moreover both even, or both odd length, then actually $\left|P_{1}\right|+\left|P_{2}\right| \geq 2^{n}-2|F|-2$.

The proof is straightforward and apart from standard induction, it uses the following results.

Proposition 6.1 (Lewinter and Widulski [12]). Let $n \geq 2$ and $u, v, w$ be distinct vertices in $Q_{n}$ such that $u$ and $v$ have the same parity opposite to the parity of $w$. Then, $Q_{n}-\{w\}$ has a Hamiltonian uv-path.

Proposition 6.2 (Hung et al. [9]). Let $n \geq 4, F \subseteq V\left(Q_{n}\right)$ such that $|F| \leq n-2$ and $F$ is not monopartite, and let $u, v \in V\left(Q_{n}\right) \backslash F$ be distinct vertices. Then, $Q_{n}-F$ has an uv-path of length at least $2^{n}-2|F|$.

In the following two lemmas we start with dimensions $n=3$ and $n=4$. Note that Lemma 6.3 is needed for Lemma 6.4, whereas Lemma 6.4 serves us as a base of induction for Theorem 1.5.

Lemma 6.3. For every set $F$ of at most 1 vertex of $Q_{3}$, there exists a long $F$-free $A B$-routing in $Q_{3}$ between every two disjoint sets $A, B \subseteq V\left(Q_{3}\right) \backslash F$ such that $|A|=|B|=2$ and $A \cup B$ is not monopartite.

Proof. It is trivial to verify the statement by inspection of all cases. First, consider all possible sets $A, B$ in case $F=\emptyset$ when we search for $A B$-routing $P_{1}, P_{2}$ in $Q_{3}$ such that $\left|P_{1}\right|+\left|P_{2}\right| \geq 5$. Then, consider the case $|F|=1$ when we need $\left|P_{1}\right|+\left|P_{2}\right| \geq 3$.

Note that the disjointness of the sets $A$ and $B$ is necessary in Lemma 6.3. Indeed, for $A=\{001,110\}, B=\{111,110\}$, and $F=\{000\}$, observe that there is no path between 001 and 111 in $Q_{3}-\{000,110\}$ of length at least 3, and consequently, no long $F$-free $A B$-routing in $Q_{3}$.

Lemma 6.4. For every set $F$ of at most 1 vertex of $Q_{4}$, there exists a long $F$-free $A B$-routing in $Q_{4}$ between every two different sets $A, B \subseteq V\left(Q_{4}\right) \backslash F$ such that $|A|=|B|=2$ and $A \cup B$ is not monopartite.

Proof. Case 1: First, we consider the case when $A=\{u, v\}$ and $B=\{x, v\}$ intersect at some vertex $v$. Then, we can treat $v$ as a new faulty vertex in the set $F^{\prime}=F \cup\{v\}$, so it suffices to find an $u x$-path in $Q_{4}-F^{\prime}$ of length at least $2^{4}-2\left|F^{\prime}\right|-1$. If $u, x$ are of opposite parity, such path exists by Corollary 1.3. Now $u$ and $x$ are of the same parity.

If $F^{\prime}=\{v\}$, then the requested $u x$-path exists by Proposition 6.1 since $A \cup B=\{u, x, v\}$ is not monopartite. Now we have $F^{\prime}=\{f, v\}$. If $f$ and $v$ have opposite parity, then the requested path exists by Proposition 6.2. 
Since $A \cup B$ is not monopartite, it remains to consider the case when $f$ and $v$ have the same parity opposite to the parity of $u$ and $x$. We split $Q_{4}$ into $Q_{L}$ and $Q_{R}$ so that $f$ and $v$ are in separate subcubes, say $F_{L}^{\prime}=\{f\}$ and $F_{R}^{\prime}=\{v\}$, and we distinguish two subcases.

Subcase (i): If vertices $u, x$ are in the same subcube, say $u, x \in V\left(Q_{L}\right)$, then from Proposition 6.1 we obtain $u x$-path $P_{L}$ in $Q_{L}-F_{L}^{\prime}$ of length 6 . Let $a b$ be an edge of $P_{L}$ such that $a_{R}, b_{R} \neq v$. From Corollary 1.3 we obtain $a_{R} b_{R}$-path $P_{R}$ in $Q_{R}-F_{R}^{\prime}$ of length 5 . After interconnecting $P_{R}$ and $P_{L}-a b$ by edges $a a_{R}, b b_{R}$ we get the desired $u x$-path in $Q_{4}-F^{\prime}$ of length $12 \geq 2^{4}-2\left|F^{\prime}\right|-1$.

Subcase (ii): Now vertices $u, x$ are in different subcubes, say $x \in V\left(Q_{L}\right)$ and $u \in V\left(Q_{R}\right)$. We choose a vertex $a \in V\left(Q_{L}\right)$ with the opposite parity than $u, a \neq f$, and $a_{R} \neq u$. Note that $a \neq x$ and $a_{R} \neq v$. From Corollary 1.3 we obtain $a x$-path $P_{L}$ in $Q_{L}-F_{L}^{\prime}$ of length 5 , and from Proposition 6.1 we obtain $u a_{R}$-path $P_{R}$ in $Q_{R}-F_{R}^{\prime}$ of length 6 . By interconnecting these paths with the edge $a a_{R}$ we obtain the desired $u x$-path in $Q_{4}-F^{\prime}$ of length $12 \geq 2^{4}-2\left|F^{\prime}\right|-1$.

Case 2: Second, we consider the case when $A=\{u, v\}$ and $B=\{x, y\}$ are disjoint. Then, we split $Q_{4}$ into $Q_{L}$ and $Q_{R}$ so that $x, y$ are in different subcubes, say $x \in V\left(Q_{L}\right)$ and $y \in V\left(Q_{R}\right)$, and we distinguish two subcases depending on the vertices of $A$.

Subcase ( $i)$ : If vertices $u, v$ are in the same subcube, say $A \subseteq V\left(Q_{L}\right)$, we choose a vertex $a \in V\left(Q_{L}\right) \backslash F_{L}$ with the same parity as $y, a_{R} \notin F_{R}$, and $a \notin\{u, v, x\}$. Note that such vertex exists, since there are 4 candidate vertices in $Q_{L}$ with the same parity as $y$, the set $F$ blocks at most one of them, and the set $\{u, v, x\}$ blocks at most two of them, otherwise $A \cup B$ would be monopartite. For a set $B^{\prime}=\{x, a\}$ it follows that $A, B^{\prime}$ are disjoint and $A \cup B^{\prime}$ is not monopartite. Hence by Lemma 6.3 , there is an $A B^{\prime}$-routing $P_{1}^{\prime}, P_{2}^{\prime}$ in $Q_{L}-F_{L}$ such that $\left|P_{1}^{\prime}\right|+\left|P_{2}^{\prime}\right| \geq 2^{3}-2\left|F_{L}\right|-3$. Assume that $a$ is the endvertex of the path $P_{1}^{\prime}$. By Corollary 1.3 , there is an $a_{R} y$-path in $Q_{R}-F_{R}$ of length at least $2^{3}-2\left|F_{R}\right|-1$ since $a_{R}$ and $y$ have opposite parity. By interconnecting $P_{1}^{\prime}$ and $P_{R}$ with the edge $a a_{R}$, we obtain $A B$-routing $P_{1}, P_{2}^{\prime}$ in $Q_{4}-F$ such that $\left|P_{1}\right|+\left|P_{2}^{\prime}\right|=\left|P_{1}^{\prime}\right|+\left|P_{R}\right|+1+\left|P_{2}^{\prime}\right| \geq 2^{4}-2|F|-3$.

Subcase (ii): Now vertices $u, v$ are in different subcubes, say $u \in V\left(Q_{L}\right)$ and $v \in V\left(Q_{R}\right)$. If $u$ and $x$, or $v$ and $y$ are of opposite parity, then from Corollary 1.3 we obtain a long $F_{L}$-free $u x$-path $P_{L}$ in $Q_{L}$ and a long $F_{R}$-free $v y$-path $P_{R}$ in $Q_{R}$ such that $\left|P_{L}\right|+\left|P_{R}\right| \geq 2^{4}-2|F|-3$. Hence $P_{L}, P_{R}$ is a long $F$-free $A B$-routing in $Q_{4}$.

Since $A \cup B$ is not monopartite, it remains to consider the case when $u$ and $x$ have the same parity opposite to the parity of $v$ and $y$. We choose two vertices $a, b \in V\left(Q_{L}\right) \backslash F_{L}$ with the same parity opposite to the parity of $u$, and $a_{R}, b_{R} \notin F_{R}$. Note that such vertices exist since there are 4 candidate vertices in $Q_{L}$ with the parity opposite to $u$ and the set $F$ blocks at most one of them. It follows that $A_{L}=\{u, x\}, B_{L}=\{a, b\}$ are disjoint and $A_{L} \cup B_{L}$ is not monopartite. Hence, by Lemma 6.3 there is a long $F_{L}$-free $A_{L} B_{L}$-routing $P_{1}^{\prime}, P_{2}^{\prime}$ in $Q_{L}$. Moreover, since both paths $P_{1}^{\prime}, P_{2}^{\prime}$ have odd length, we have $\left|P_{1}^{\prime}\right|+\left|P_{2}^{\prime}\right| \geq 2^{3}-2\left|F_{L}\right|-2$. Assume that the $A_{L} B_{L^{-}}$-routing joins the vertex $u$ with $b$, otherwise we switch the roles of $a$ and $b$ in what follows. By the definition of $a, b$, the sets $A_{R}=\left\{b_{R}, v\right\}, B_{R}=\left\{a_{R}, y\right\}$ are disjoint and $A_{R} \cup B_{R}$ is not monopartite. Hence, by Lemma 6.3 there is a long $F_{R}$-free $A_{R} B_{R}$-routing $P_{3}^{\prime}, P_{4}^{\prime}$ in $Q_{R}$. By interconnecting $P_{1}^{\prime}, P_{2}^{\prime}$ and $P_{3}^{\prime}, P_{4}^{\prime}$ with edges $a a_{R}, b b_{R}$ we obtain $A B$-routing $P_{1}, P_{2}$ in $Q_{4}-F$ such that $\left|P_{1}\right|+\left|P_{2}\right|=\left|P_{1}^{\prime}\right|+\left|P_{2}^{\prime}\right|+\left|P_{3}^{\prime}\right|+\left|P_{4}^{\prime}\right|+2 \geq 2^{4}-2|F|-2$.

Now we are ready to prove Theorem 1.5, which says that for every set $F$ of at most $n-3$ vertices in $Q_{n}$ and $n \geq 4$, there exists a long $F$-free $A B$-routing in $Q_{n}$ between every two different sets $A, B \subseteq V\left(Q_{n}\right) \backslash F$ such that $|A|=|B|=2$ and $A \cup B$ is not monopartite. 
Proof of Theorem 1.5. We proceed by induction on the dimension $n$. For $n=4$ we apply Lemma 6.4. Now assume $n \geq 5$.

First, we split $Q_{n}$ into $Q_{L}$ and $Q_{R}$ such that we separate two arbitrarily chosen faulty vertices from $F$ if $|F| \geq 2$, otherwise we split $Q_{n}$ arbitrarily. It follows that $\left|F_{L}\right|,\left|F_{R}\right| \leq n-4$. Thus, we may apply induction both in $Q_{L}$ and $Q_{R}$. We consider the following cases.

Case 1: If both $A, B$ are in one subcube, say $A, B \subseteq V\left(Q_{L}\right)$, then by induction, there is a long $F_{L}$-free $A B$-routing $P_{1}^{\prime}, P_{2}^{\prime}$ in $Q_{L}$. Let $a b$ be an edge of $P_{1}^{\prime}$ or $P_{2}^{\prime}$, such that $a_{R}, b_{R} \notin F_{R}$. Such edge exists, otherwise $2^{n-1}-2\left|F_{L}\right|-3 \leq\left|P_{1}^{\prime}\right|+\left|P_{2}^{\prime}\right| \leq 2\left|F_{R}\right|$, which yields a contradiction $2^{n-1}-3 \leq 2|F| \leq 2 n-6$ for $n \geq 5$. From Corollary 1.3 we obtain an $a_{R} b_{R}$-path $P_{R}$ in $Q_{R}-F_{R}$ of length $2^{n-1}-2\left|F_{R}\right|-1$ since $a_{R}$ and $b_{R}$ have different parity. After interconnecting $P_{R}$ and $P_{1}^{\prime}$ or $P_{2}^{\prime}$ with the edges $a a_{R}, b b_{R}$ we get the $A B$-routing $P_{1}, P_{2}$ in $Q_{n}-F$ such that $\left|P_{1}\right|+\left|P_{2}\right|=\left|P_{1}^{\prime}\right|+\left|P_{2}^{\prime}\right|+\left|P_{R}\right|+1 \geq 2^{n}-2|F|-3$.

Case 2: If $A$ is in one subcube and $B$ in the other subcube, say $A=\{u, v\} \subseteq V\left(Q_{L}\right)$ and $B=\{x, y\} \subseteq V\left(Q_{R}\right)$, we distinguish two subcases.

Subcase (i): If $u$ and $v$ have different parity, then from Corollary 1.3 we obtain an $u v$-path $P_{L}$ in $Q_{L}-F_{L}$ of length at least $2^{n-1}-2\left|F_{L}\right|-1$. Let $a b$ be an edge of $P_{L}$ such that $A^{\prime}=\left\{a_{R}, b_{R}\right\}$ is disjoint with $F_{R}$ and $A^{\prime} \neq B$. Such edge exists, otherwise $\left|P_{R}\right| \leq 2\left|F_{R}\right|+1$, which yields a contradiction $2^{n-1}-2 \leq 2|F| \leq 2 n-6$ for $n \geq 5$. Since $A^{\prime} \cup B$ is not monopartite, there is a long $F_{R}$-free $A^{\prime} B$-routing $P_{1}^{\prime}, P_{2}^{\prime}$ in $Q_{R}$. By interconnecting $P_{L}-a b$ and $P_{1}^{\prime}, P_{2}^{\prime}$ with the edges $a a_{R}, b b_{R}$, we get an $A B$-routing $P_{1}, P_{2}$ in $Q_{n}-F$ such that $\left|P_{1}\right|+\left|P_{2}\right|=\left|P_{L}\right|+\left|P_{1}^{\prime}\right|+\left|P_{2}^{\prime}\right|+1 \geq 2^{n}-2|F|-3$.

Subcase (ii): Now $u$ and $v$ are of the same parity. We choose vertices $B^{\prime}=\{a, b\} \subseteq$ $V\left(Q_{L}\right) \backslash F_{L}$ of the same parity opposite to the parity of $u$ such that $A^{\prime}=\left\{a_{R}, b_{R}\right\}$ is disjoint with $F_{R}$. Such vertices exists, since there are $2^{n-2}$ candidates in $Q_{L}$ with parity opposite to the parity of $u$, and at most $n-3$ of them are blocked by $F$. Clearly, $A \neq B^{\prime}$ and $A \cup B^{\prime}$ is not monopartite. Thus, there is a long $F_{L}$-free $A B^{\prime}$-routing $P_{1}^{\prime}, P_{2}^{\prime}$ in $Q_{L}$. Moreover, since both $P_{1}^{\prime}, P_{2}^{\prime}$ have odd length, we have $\left|P_{1}^{\prime}\right|+\left|P_{2}^{\prime}\right| \geq 2^{n-1}-2\left|F_{L}\right|-2$. In the other subcube $Q_{R}$, at least one vertex of $B=\{x, y\}$ has the opposite parity to the parity of $a_{R}, b_{R}, u$, and $v$. It follows that $A^{\prime} \neq B$ and $A^{\prime} \cup B$ is not monopartite, and hence, there is a long $F_{R}$-free $A^{\prime} B$-routing $P_{3}^{\prime}, P_{4}^{\prime}$ in $Q_{R}$. By interconnecting $P_{1}^{\prime}, P_{2}^{\prime}$ and $P_{3}^{\prime}, P_{4}^{\prime}$ with edges $a a_{R}, b b_{R}$ we get an $A B$-routing $P_{1}, P_{2}$ such that $\left|P_{1}\right|+\left|P_{2}\right|=\left|P_{1}^{\prime}\right|+\left|P_{2}^{\prime}\right|+\left|P_{3}^{\prime}\right|+\left|P_{4}^{\prime}\right|+2 \geq 2^{n}-2|F|-3$.

Case 3: If $A$ is one subcube, and $B$ in both subcubes, say $A=\{u, v\} \subseteq V\left(Q_{L}\right), x \in V\left(Q_{L}\right)$, $y \in V\left(Q_{R}\right)$, then we proceed similarly as in Case 2, Subcase $(i)$ of Lemma 6.4. We choose a vertex $a \in V\left(Q_{L}\right) \backslash F_{L}$ with the same parity as $y, a_{R} \notin F_{R}$, and $a \notin\{u, v, x\}$. Note that such vertex exists, since there are $2^{n-2}$ candidate vertices in $Q_{L}$ with the same parity as $y$, the faulty vertices block at most $n-3$ of them, the set $\{u, v, x\}$ blocks at most 3 of them, and $2^{n-2}-(n-3)-3 \geq 1$ for $n \geq 5$. For a set $B^{\prime}=\{x, a\}$ it follows that $A, B^{\prime}$ are disjoint and $A \cup B^{\prime}$ is not monopartite. Hence by induction, there is an $A B^{\prime}$-routing $P_{1}^{\prime}, P_{2}^{\prime}$ in $Q_{L}-F_{L}$ such that $\left|P_{1}^{\prime}\right|+\left|P_{2}^{\prime}\right| \geq 2^{n-1}-2\left|F_{L}\right|-3$. Assume that $a$ is the endvertex of the path $P_{1}^{\prime}$. By Corollary 1.3, there is an $a_{R} y$-path in $Q_{R}-F_{R}$ of length at least $2^{n-1}-2\left|F_{R}\right|-1$ since $a_{R}$ and $y$ have opposite parity. By interconnecting $P_{1}^{\prime}$ and $P_{R}$ with the edge $a a_{R}$, we obtain $A B$-routing $P_{1}, P_{2}^{\prime}$ in $Q_{n}-F$ such that $\left|P_{1}\right|+\left|P_{2}^{\prime}\right|=\left|P_{1}^{\prime}\right|+\left|P_{R}\right|+1+\left|P_{2}^{\prime}\right| \geq 2^{n}-2|F|-3$.

Case 4: If $A, B$ are both subcubes, say $u, x \in V\left(Q_{L}\right)$ and $v, y \in V\left(Q_{R}\right)$, then we proceed similarly as in Case 2, Subcase (ii) of Lemma 6.4. If $u$ and $x$, or $v$ and $y$ are of opposite parity, then from Corollary 1.3 we obtain a long $F_{L}$-free $u x$-path $P_{L}$ in $Q_{L}$ and a long $F_{R}$-free 
$v y$-path $P_{R}$ in $Q_{R}$ such that $\left|P_{L}\right|+\left|P_{R}\right| \geq 2^{n}-2|F|-3$. Hence $P_{L}, P_{R}$ is a long $F$-free $A B$-routing in $Q_{n}$.

Since $A \cup B$ is not monopartite, it remains to consider the case when $u$ and $x$ have the same parity opposite to the parity of $v$ and $y$. We choose two vertices $a, b \in V\left(Q_{L}\right) \backslash F_{L}$ with the same parity opposite to the parity of $u$, and $a_{R}, b_{R} \notin F_{R}$. Note that such vertices exist since there are $2^{n-2}$ candidate vertices in $Q_{L}$ with the parity opposite to the parity of $u$, the faulty vertices block at most $n-3$ of them, and $2^{n-2}-(n-3) \geq 2$ for $n \geq 5$. It follows that $A_{L}=\{u, x\}, B_{L}=\{a, b\}$ are disjoint and $A_{L} \cup B_{L}$ is not monopartite. Hence, by induction there is a long $F_{L}$-free $A_{L} B_{L}$-routing $P_{1}^{\prime}, P_{2}^{\prime}$ in $Q_{L}$. Moreover, since both paths $P_{1}^{\prime}, P_{2}^{\prime}$ have odd length, we have $\left|P_{1}^{\prime}\right|+\left|P_{2}^{\prime}\right| \geq 2^{n-1}-2\left|F_{L}\right|-2$. Assume that the $A_{L} B_{L}$-routing joins the vertex $u$ with $b$, otherwise we switch the roles of $a$ and $b$ in what follows. By the definition of $a, b$, the sets $A_{R}=\left\{b_{R}, v\right\}, B_{R}=\left\{a_{R}, y\right\}$ are disjoint and $A_{R} \cup B_{R}$ is not monopartite. Hence, by induction there is a long $F_{R}$-free $A_{R} B_{R}$-routing $P_{3}^{\prime}, P_{4}^{\prime}$ in $Q_{R}$. By interconnecting $P_{1}^{\prime}, P_{2}^{\prime}$ and $P_{3}^{\prime}, P_{4}^{\prime}$ with edges $a a_{R}, b b_{R}$ we obtain $A B$-routing $P_{1}, P_{2}$ in $Q_{n}-F$ such that $\left|P_{1}\right|+\left|P_{2}\right|=\left|P_{1}^{\prime}\right|+\left|P_{2}^{\prime}\right|+\left|P_{3}^{\prime}\right|+\left|P_{4}^{\prime}\right|+2 \geq 2^{n}-2|F|-2$.

Finally, we prove Corollary 1.6 that says for every set $F$ of at most $n-2$ vertices of $Q_{n}$ and $n \geq 4$, the graph $Q_{n}-F$ has an $u v$-path of length at least $2^{n}-2|F|-1$ for every two vertices $u, v \in V\left(Q_{n}\right) \backslash F$ such that $F \cup\{u, v\}$ is not monopartite.

Proof of Corollary 1.6. If $F=\emptyset$, then $u$ and $v$ have opposite parity, and the statement follows from a well-known fact that $Q_{n}$ contains a Hamiltonian path between every two vertices of opposite parity. Otherwise, there exists $f \in F$ such that $\{u, v, f\}$ is not monopartite. Applying Theorem 1.5 for $A=\{u, f\}, B=\{v, f\}, F^{\prime}=F \backslash\{f\}$ we obtain vertex-disjoint paths $P_{1}, P_{2}$ such that $P_{1}$ joins $u$ and $v, P_{2}$ contains only $f$, and $\left|P_{1}\right|+\left|P_{2}\right| \geq 2^{n}-2\left|F^{\prime}\right|-3$. Hence $\left|P_{1}\right| \geq 2^{n}-2|F|-1$, and $P_{1}$ is the desired path. 